\title{
Acculturation and physical activity among immigrants: a systematic review
}

\author{
Markus Gerber • Dean Barker • Uwe Pühse
}

Received: 22 February 2011 /Accepted: 10 August 2011 /Published online: 6 September 2011

(C) Springer-Verlag 2011

\begin{abstract}
Aim The acculturation process presents numerous challenges that could benefit or adversely affect immigrants' health practices. The goal of this review was to present a systematic summary of studies examining the relationship between acculturation and physical activity among immigrants and ethnic minority populations.

Subjects and methods Systematic review based on 44 original studies found in electronic databases (Psychinfo/ Psyndex, Eric, Pubmed/Medline, Web of Science, SportDiscus). Studies were eligible if they were written in English, German or French, incorporated a measure of acculturation and physical activity, exercise, or sport as independent and dependent variables, and provided statistical information to judge the level of significance. The 44 studies found included 760,242 participants. A narrative synthesis was performed. Causality of effects was examined based on seven criteria: consistency, strength of associations, specificity of effects, temporality, gradient, plausibility and experimental evidence.

Results Acculturation was associated with higher leisure time physical activity in $57 \%$ of all studies (even after controlling for potential confounds), independent of participants' gender, age and ethnic background. The main limitations of this review are that findings were not weighted for sample size and that publication biases might have contributed to an overestimation of the relationships. Conclusion Prevention programs aimed at stimulating participation in physical activity among immigrants should give priority to individuals with low acculturation levels and it
\end{abstract}

M. Gerber $(\bowtie) \cdot$ D. Barker $\cdot$ U. Pühse

Institute of Exercise and Health Science, University of Basel,

Birsstrasse 320B,

4052 Basel, Switzerland

e-mail: markus.gerber@unibas.ch should consider culturally specific beliefs and constraints. Additionally, prevention programs could make an effort to promote acculturation among immigrants. Future studies should use longitudinal designs which include objective assessments of physical activity and which use multidimensional and bidirectional definitions of acculturation.

Keywords Ethnicity Exercise · Immigrants · Immigration · Minority · Sport

\section{Introduction}

Reducing health disparities in ethnic populations is a major public health objective in many Western nations (Carvajal et al. 2002). Having a migration background or ethnic minority status, however, does not constitute a health risk per se. Sam (2006) presented several hypotheses of how migration might be related to health. First, health differences might be due to a selection effect. That is, individuals who are predisposed to health problems are those who want to emigrate (e.g. coming from poor countries, traumatic experiences). Second, the stress effect postulates that health differences might be due to the inability of immigrants to cope with the stresses associated with migration and the acculturation thereafter. Third, the healthy immigrant effect assumes that people who migrate are healthier than those who do not. And fourth, the immigrant paradox suggests that immigrants have better health than their socioeconomic background suggest, and that, while they arrive with better health than their non-immigrant counterparts, this better health deteriorates over time (e.g. with prolonged length of residence or increasing generational status). Interestingly, empirical support exists for all four hypotheses, which might be due to the fact that the circumstances 
under which migration takes place vary considerably between individuals and across ethnic groups. It is reasonable to expect that the nature of the diseases investigated has a moderating impact (e.g. immigrants coming from African countries with higher risk for infectious diseases such as Tuberculosis, and lower risks of cardiovascular diseases or cancer). Furthermore, within the framework of the 'immigrant paradox', the level of acculturation (e.g. generational status, language use) has been identified as an important influence.

Acculturation is generally defined as the merging of cultures as a result of prolonged contact between individuals and groups of different cultural backgrounds (Crespo et al. 2001; Sam 2006). Thus, acculturation is often described as the process by which immigrants adopt the attitudes, values, customs, beliefs, behaviors, and the lifestyles of a new culture and the dominant society (Abraido-Lanza et al. 2006; Lara et al. 2005). However, researchers have underlined since the 1970s that assimilation is not the only form of acculturation, but that individuals might adopt at least four different approaches towards acculturation including assimilation, biculturalism, separation, marginalization (Berry 1970). Bicultural orientation is characterized as seeking relationships with the host society, while simultaneously maintaining the heritage culture and identity. In contrast, separation is characteristic for individuals who do seek contacts outside their own minority ethnicity, whereas marginalization describes individuals who identify neither with their heritage nor their host society (Berry 2006). Moreover, acculturation is a complex concept that no single factor can adequately describe. When detailed information on culture and attitude is not available, researchers often use more accessible indicators such as place of birth, length of residence in the receiving country, and language preference, either independently or in combination, as proxy measures of acculturation (Lara et al. 2005).

Scientists have argued that acculturation may influence health practices as the acculturation process presents numerous challenges and life changes that could potentially benefit or adversely affect the health behaviors of immigrants as well as subsequent immigrant generations (Abraido-Lanza et al. 2006; Crespo et al. 2001). In accordance with this idea, several studies conducted in the USA have found negative relationships between acculturation and health practices even after adjustment for social disadvantage confounders. That is, research with Hispanic and Asian-American adolescents has implicated acculturation to the US as a risk factor for unhealthy behaviors. For instance, patterns of alcohol abuse, tobacco use, eating disorders, and unhealthy dietary practices were stronger among Mexican Americans who were more acculturated than among those less acculturated (e.g. De La Rosa et al. 2000; Unger et al. 2004).
Regarding the relationship between acculturation and physical activity, Unger et al. (2004) stated that relatively little research has been done. This, however, has changed as research efforts in this area have increased considerably since the turn of the millennium. Based on epidemiological studies showing that ethnic majority populations are generally more active than ethnic minority groups (Epstein et al. 2008; Yeager et al. 1993), researchers have assumed that with increasing acculturation, the level of physical activity approaches the level found in the host population (Hosper et al. 2008). That is, physical activity might differ from other health behaviors and health outcomes, where immigrants have been shown to have a significant advantage over their non-immigrant counterparts (Singh et al. 2008).

Generally, research on acculturation goes beyond descriptive studies which examined ethnic/race differences in physical activity in the sense that acculturation is considered as a potential factor to explain discrepancies. With regard to ethnic differences, previous research in Western countries has shown that (1) ethnic minorities mostly report lower levels of leisure time physical activity (e.g. Crespo et al. 2000; Sagatun et al. 2008), (2) ethnic minorities meet recommendations for weekly leisure-time physical activity to a lower extent than the majority population (e.g. Schoenborn et al. 2004), (3) differences are particularly marked among minority women (e.g. Byrd-Williams et al. 2007) and (4) physical activity of ethnic minorities is influenced by a number of individual-level factors such as socioeconomic status, education, and marital status (Evenson et al. 2003).

Since regular physical activity is an important health resource that reduces health costs and contributes to individual health and well-being (Faulkner and Taylor 2005; Sallis and Owen 1999), it is not surprising that increasing the level of physical activity among ethnic minorities has been identified as an important public health target. Against this background, the present article intends to provide a systematic review over past studies examining the relationship between acculturation and physical activity among immigrants and ethnic minority populations. The following questions will be addressed: First, does past research support a positive influence of acculturation on daily and recreational physical activity? Second, is the evidence independent of the host society in which the study took place, the age, gender and ethnicity of the participants, the date and source of publication, and the methods of data analysis? Third, how strong are the associations between acculturation and physical activity? Fourth, do the findings depend on the measurement of physical activity and acculturation? Fifth, does the temporal sequence of the data allow the establishing of cause-effect relationships? Sixth, is there evidence for a dose-response relationship? 
Seventh, can the relationships be maintained if the analyses are controlled for possible confounds? Eighth, does experimental evidence support a causal influence of acculturation on levels of physical activity?

\section{Methods}

\section{Review method}

The present review is based on narrative synthesis. As outlined in the introduction, acculturation is a complex phenomenon. Moreover, researchers have shown that prolonged length of residence in a host country does not necessarily lead to assimilation, and that individuals might prefer different approaches towards acculturation including biculturalism, separation or marginalization. Given the bidirectional/orthogonal nature of the construct, using a meta-analytic procedure to establish an effect-size for the strength of the relationship between acculturation and physical activity was not appropriate.

Databases searched for this review include Psychinfo/ Psyndex, Eric, Pubmed/Medline, Web of Science, and SportDiscus. The last search was carried out in April 2011. We combined the following keywords to search the literature: physical activity, exercise or sport (in the title), and acculturation (in the abstract). In addition to that, citation lists from papers were cross-referenced. Articles selected for inclusion in this review met the following criteria: (1) they were either written in English, German or French; (2) they incorporated (a) a measure of acculturation (e.g. length of residence, language use, adoption of values) as an independent variable, and (b) one or several dependent variables assessing the level of physical activity, exercise, or sport participation as a dependent variable; (3) they provided sufficient statistical information to conclude whether the associations were significant or not; (4) they used measures of leisure-time physical activity (e.g. exercise, sports) and daily physical activity (e.g. household, gardening). Studies that assessed occupational physical activity were excluded because it can be assumed that occupational physical activity is predominantly influenced by type of occupation, and that these activities can be less influenced by individual choice. Thus, the relationship between acculturation and daily and leisure-time physical activity seems more relevant from a public health point of view (BFS 2006).

Our basic hypothesis was that leisure-time physical activity would increase with increasing acculturation. To test this hypothesis, we differentiated between studies that supported, partly supported or did not support significant relationships between acculturation and physical activity. Commonly, multiple indicators were used to assess accul- turation and physical activity, which is why many studies provided more than one single statistical coefficient. Furthermore, many studies provided several coefficients to compare groups with distinct levels of acculturation against a reference group. To deal with this difficulty, we classified studies with more than $66.6 \%$ of significant main effects as 'supportive'. If $33.3-66.6 \%$ of the analyses supported a main effect, studies were considered 'partly supportive'. If less than $33.3 \%$ of the statistics were significant or provided findings non-compliant with our hypothesis, the studies were classified as 'non-supportive'. Although these cut-off criteria might seem arbitrary, we argue that using three equally broad categories allows the most balanced interpretation of the findings. Additionally, we have indicated for each study the ratio of significant coefficient in relation to the total number of tests of significance (Table 1). In some studies, unadjusted and adjusted analyses were provided. In this case, the analyses with the higher level of adjustment were used to establish the significance of effects. Despite using the 5\% level of significance as a cut-off criterion, this review does not purely rely on a votecounting procedure. Vote-counting may be a problematic approach that may entail misleading conclusions (e.g. no validity concerns, reliance of sample size). Accordingly, we employed Hill's (1965) criteria (consistency, strength of associations, specificity of effects, temporality, gradient, plausibility and experimental evidence) in order to determine more solidly to what degree the association between acculturation and physical activity can be regarded as causal.

Specifically, Hill (1965) argued that an association between constructs can be viewed as more evidence-based if it has been observed repeatedly over time by different researchers, in different places, in different circumstances and times, and by using different methodological approaches. Accordingly, consistency refers to the issue of external validity, that is, to the degree of generalizability of effects across individuals and methodologies. Thus, we examined whether supportive findings were found (1) in different countries, (2) in different age groups, (3) in female versus male subjects, (4) in different ethnic minority groups, (5) in different moments in time, (6) including populations with small versus large sample sizes, and (7) using different methods of data analysis. Moreover, Hill (1965) emphasized that the strength of association is an important indicator of causality because it shifts attention from interpreting levels of significance towards meaning and relevance of empirical data. Additionally, specificity strengthens the plausibility of cause-effect relationships. As an example, Hill (1965) argued that specificity of effects refers to whether a specific disease (e.g. lung cancer) is limited to specific conditions thought to be responsible for this disease (e.g. smoking). Referring to specificity, we 
Table 1 Overview of studies classified as supportive, partly supportive and non-supportive

\begin{tabular}{|c|c|c|c|c|c|}
\hline \multicolumn{2}{|l|}{ Supportive studies } & \multicolumn{2}{|l|}{ Partly supportive studies } & \multicolumn{2}{|l|}{ Non-supportive studies } \\
\hline Pérez-Stable et al. 1994 & $100.0 \%$ & Lee et al. 2000 & $37.5 \%$ & Cantero et al. 1999 & $0.0 \%$ \\
\hline Crespo et al. 2001 & $66.7 \%$ & Richman et al. 2000 & $50.0 \%$ & Carvajal et al. 2002 & $0.0 \%$ \\
\hline Song et al. 2004 & $66.7 \%$ & Evenson et al. 2004 & $44.4 \%$ & Gordon-Larsen et al. 2003 & $16.7 \%$ \\
\hline Hosper et al. 2007 & $100 \%$ & Abraido-Lanza et al. 2005 & $50.0 \%$ & Wilbur et al. 2003 & $0.0 \%$ \\
\hline Corral and Landrine 2008 & $100 \%$ & Dergance et al. 2005 & $40.0 \%$ & Unger et al. 2004 & $0.0 \%$ \\
\hline Neighbors et al. 2008 & $75.0 \%$ & Kandula and Lauderdale 2005 & $44.4 \%$ & Dawson et al 2005 & $25 \%$ \\
\hline Singh et al. 2008 & $66.7 \%$ & Berrigan et al. 2006 & $50.0 \%$ & Slattery et al. 2006 & $20 \%$ \\
\hline Liu et al. 2009 & $66.7 \%$ & Tremblay et al. 2006 & $64.3 \%$ & Crespo et al. 2006 & $0.0 \%$ \\
\hline \multirow[t]{11}{*}{ Dogra et al. 2010} & $75.0 \%$ & Wolin et al. 2006 & $37.5 \%$ & Fitzgerald et al. 2006 & $0.0 \%$ \\
\hline & & Allen et al. 2007 & $50.0 \%$ & Marquez et al. 2006 & $0.0 \%$ \\
\hline & & Pichon et al. 2007 & $50.0 \%$ & Masel et al. 2006 & $0.0 \%$ \\
\hline & & Choi et al. 2008 & $50.0 \%$ & Yang et al. 2007 & $0.0 \%$ \\
\hline & & Hofstetter et al. 2008 & $50.0 \%$ & Mainous et al. 2008 & $0.0 \%$ \\
\hline & & Hosper et al. 2008 & $50.0 \%$ & Martinez et al. 2008 & $11.1 \%$ \\
\hline & & Renzaho et al. 2008 & $51.9 \%$ & Mejean et al. 2009 & $0.0 \%$ \\
\hline & & Springer et al. 2009 & $33.3 \%$ & Chen 2009 & $0.0 \%$ \\
\hline & & & & Afable-Munsuz et al. 2010 & $21.4 \%$ \\
\hline & & & & Ghaddar et al. 2010 & $25 \%$ \\
\hline & & & & Taverno et al. 2010 & $25 \%$ \\
\hline 9 studies & & 16 studies & & 19 studies & \\
\hline
\end{tabular}

Note: Multiple samples and indicators of acculturation and leisure-time physical activity were used in most studies. Therefore, studies with $0<$ $33.3 \%$ of supportive findings were classified as non-supportive, studies with $\geq 33.3-66.6 \%$ of supportive findings as partly supportive and studies with $\geq 66.6 \%$ of supportive findings as supportive. The exact percentage of supportive findings is indicated after each study. More detailed information about the total number of analyses in each study is provided in Table 2.

examined whether positive associations between acculturation and physical activity existed independent of the physical activity and acculturation measures. Furthermore, the criterion of temporality is closely connected with issues regarding the internal validity of the study design. Following Hill (1965), temporal sequence addresses the question of "which is the cart and which the horse" (p. 297), and might hence answer whether individuals become more physically active as they get more acculturated or whether physical activity might contribute to the acculturation of individuals. As generally accepted, temporal sequence can only be tested through prospective, longitudinal or experimental studies. What Hill (1965) called a gradient is currently known as a dose-response relationship. In our review, two aspects are of particular interest, (1) whether an increase in acculturation results in linearly increasing physical activity and (2) whether physical activity increases more strongly if individuals report high scores on multiple acculturation indicators (e.g. second generation immigration status in combination with high language proficiency). The concept of plausibility refers to the question of whether particular mechanisms can convincingly explain the positive relationships between acculturation and physical activity. Therefore, we examined whether previous analyses were sufficiently controlled for potential confounds, and whether the associations are moderated by environmental factors. Finally, Hill (1965) argued that the best evidence for a causal link might come under the criterion of experimental evidence. The last question is therefore, if it is possible to observe (experimentally induced or naturally occurring) circumstances that lead to changed patterns between acculturation and physical activity.

\section{Results}

Studies included in the review

Forty-four studies dealing with the relationship between acculturation and physical activity among ethnic minority groups were found that met all the aforementioned inclusion criteria. Figure 1 provides a flow chart of how the studies were screened, assessed for eligibility, and included in the review. The review includes a total of 760,242 participants. Table 1 indicates which studies were classified as supportive, partly supportive and nonsupportive. Detailed information about the samples, sam- 
Fig. 1 Overview of the study selection process following the PRISMA Statement (Moher et al. 2009)

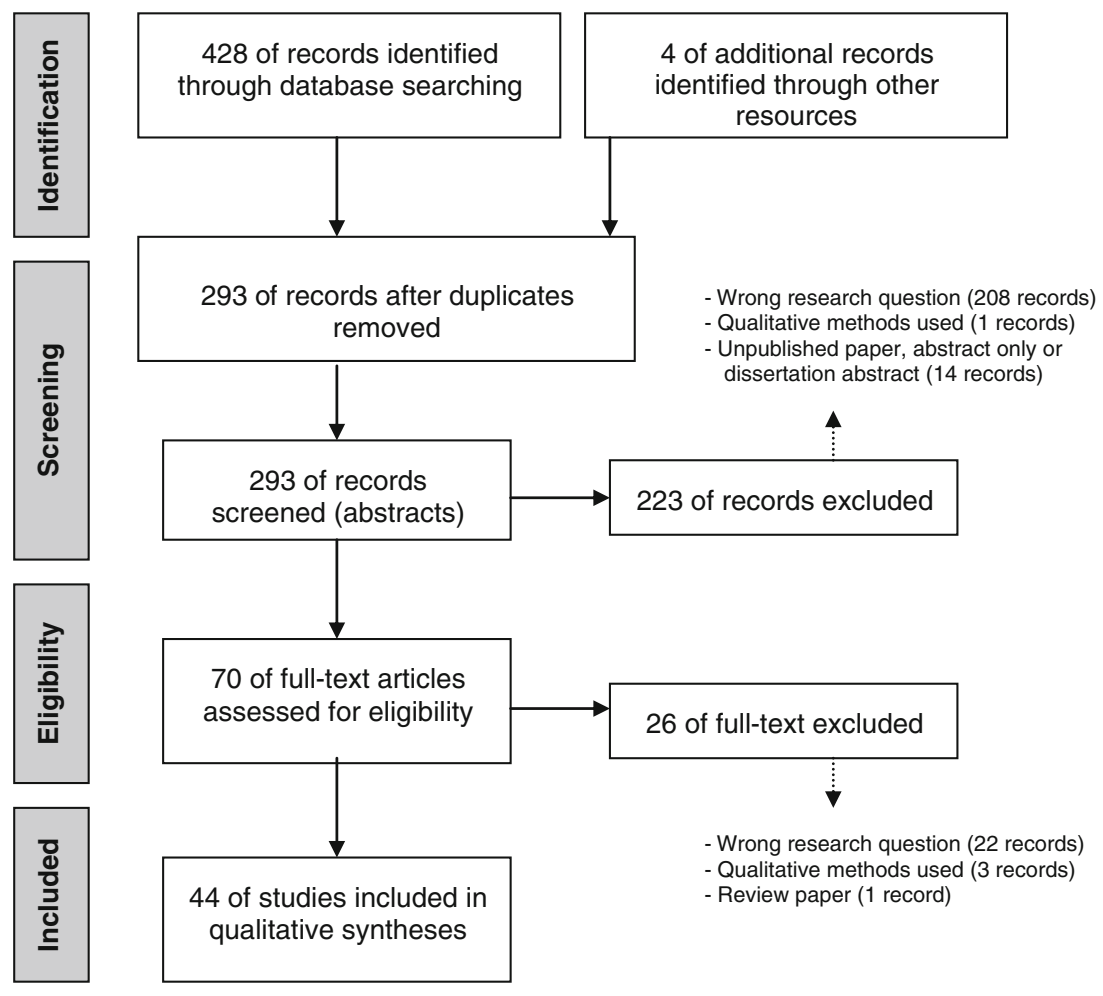

pling methods, research designs, control of confounding variables, measurements and data-analysis techniques and the results is provided in Table 2 (listed in chronological order, and alphabetical order if several studies were published in the same year). In addition, Table 2 provides the ratio of significant/total tests of significance for each single study.

Nine studies supported the hypothesis that increased acculturation is associated with higher leisure time and daily physical activity (see Table 1). In addition, 16 studies showed partial support. In contrast, 19 studies did not confirm a main effect between acculturation and physical activity. Among the non-supportive studies, four investigations provided contradicting results in the sense that higher acculturation was related to decreased physical activity (Berrigan et al. 2006; Cantero et al. 1999; Marquez and McAuley 2006; Unger et al. 2004).

\section{Criterion 1: consistency of evidence}

Location Previous studies have been carried out in the USA, Canada, Sweden, the Netherlands, Australia and France. Positive relationships between acculturation and daily/leisure time were found both in North American (eight studies supportive, 13 partly supportive), European (one study supportive, one study partly supportive) and Australian research (two studies partly supportive). Interestingly, in other parts of the world, no attempts have been made to examine this issue.
Participants' age Most research has been conducted with adults (31 studies). Only eight studies have focused on children and adolescents. Adult studies provided $61 \%$ supportive findings (six supportive, 13 studies partly supportive studies), whereas among children and adolescents $63 \%$ of prior research showed supportive results (two supportive, three partly supportive studies). Non-supportive findings were found in studies with younger children (Crespo et al. 2006; Martinez et al. 2008; Taverno et al. 2010; Unger et al. 2004) and older adolescents (Carvajal et al. 2002; Gordon-Larsen et al. 2002). Research with elderly people is rare, but indicates that acculturation is less influential in older age (Cantero et al. 1999; Masel et al. 2006; Mejean et al. 2009), although positive findings were reported with elderly Mexicans (Dergance et al. 2005) and Koreans (Richman et al. 2000).

Gender differences Eight studies exclusively focused on female samples (Cantero et al. 1999; Choi et al. 2008; Evenson et al. 2004; Fitzgerald et al. 2006; Hosper et al. 2008; Pichon et al. 2007; Slattery et al. 2006; Wilbur et al. 2003), whereas one study only focused on men (Mejean et al. 2009). All other investigations used mixed samples. Among female studies, $50 \%$ of the investigations were partly supportive, whereas $50 \%$ of the studies did not find significant relationships. The only study focusing on males was not supportive (Mejean et al. 2009). Most investigations with mixed samples did not identify major differences between men and women. Gender sensitive analyses, 


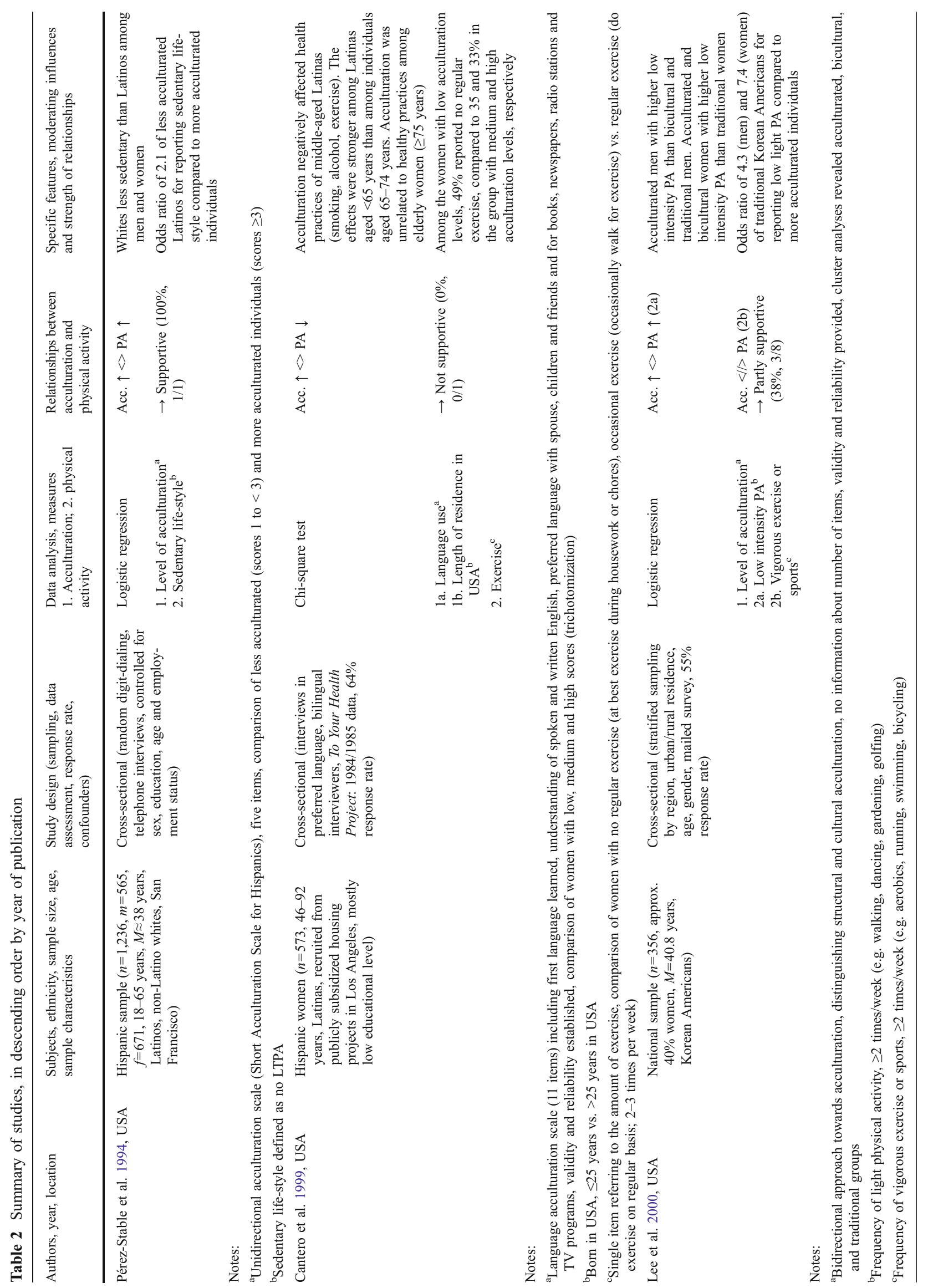



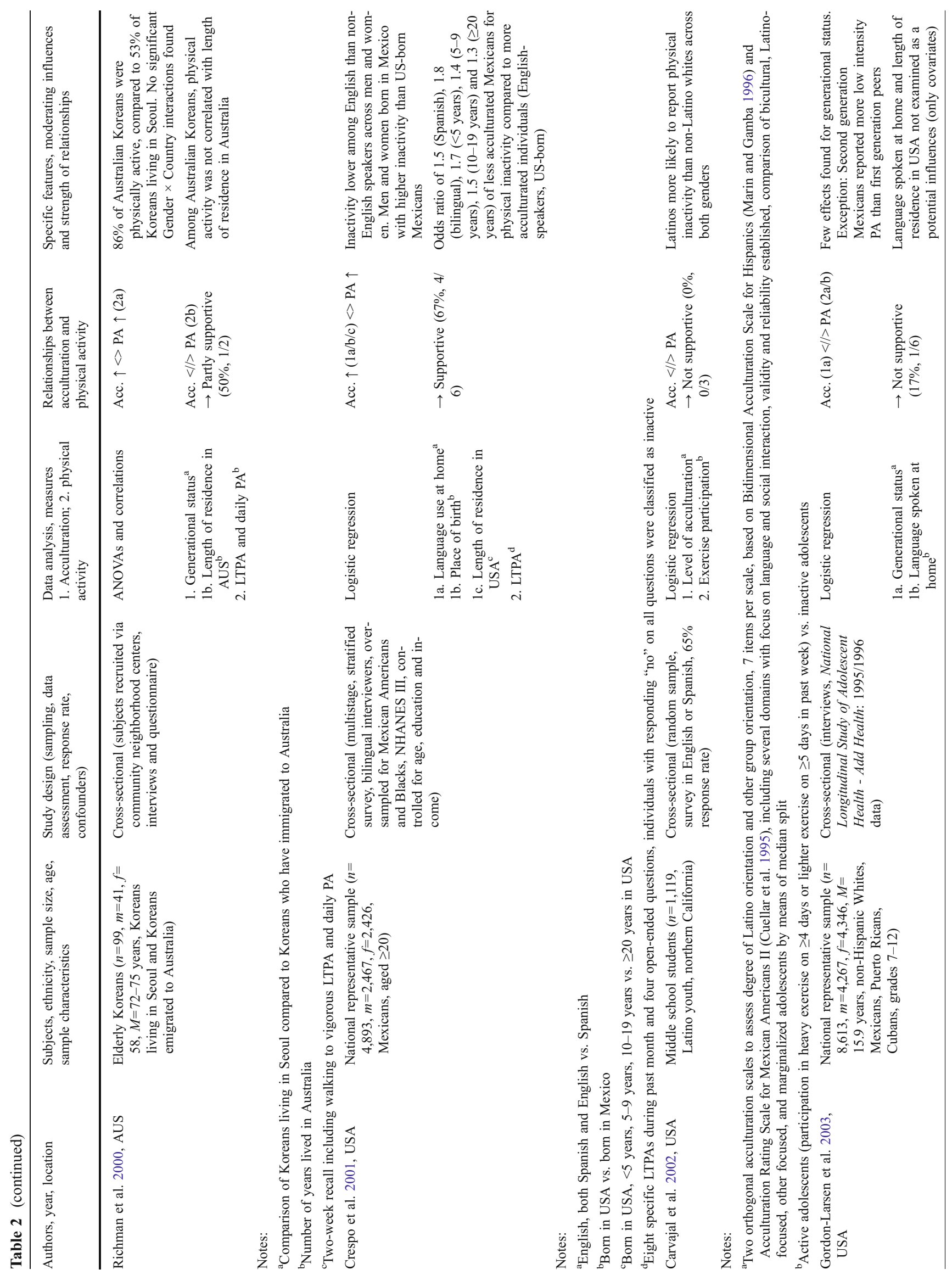


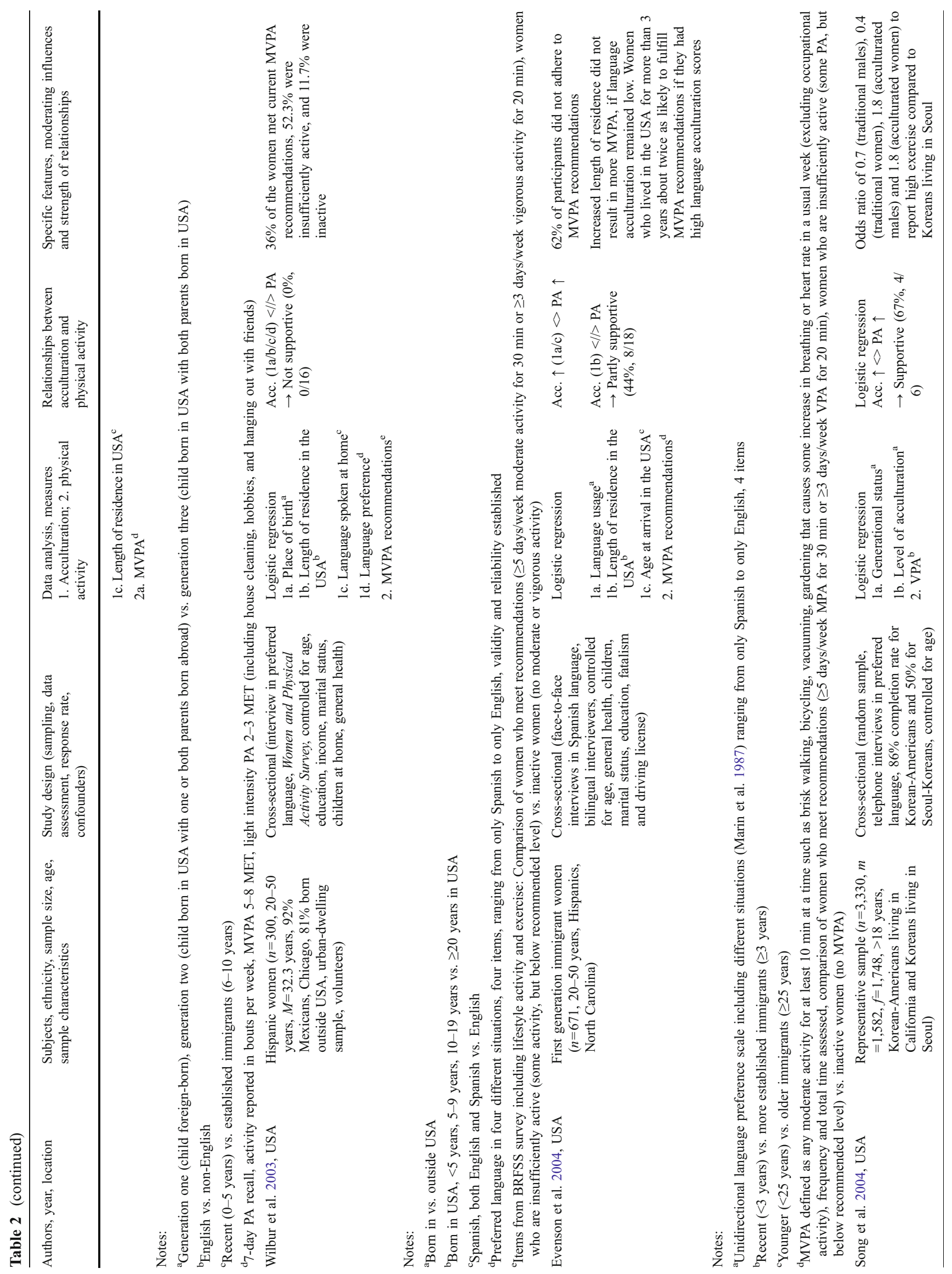




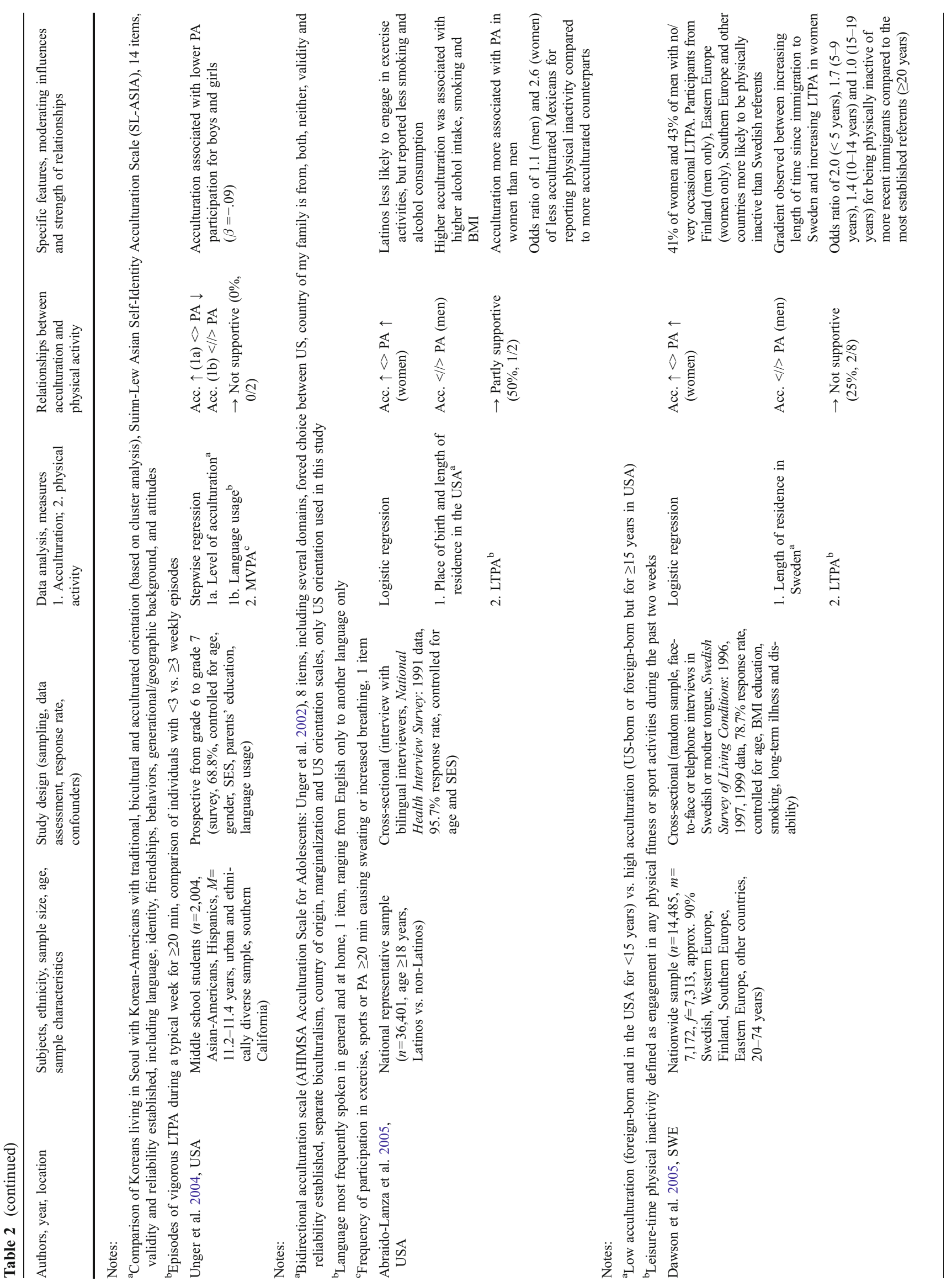




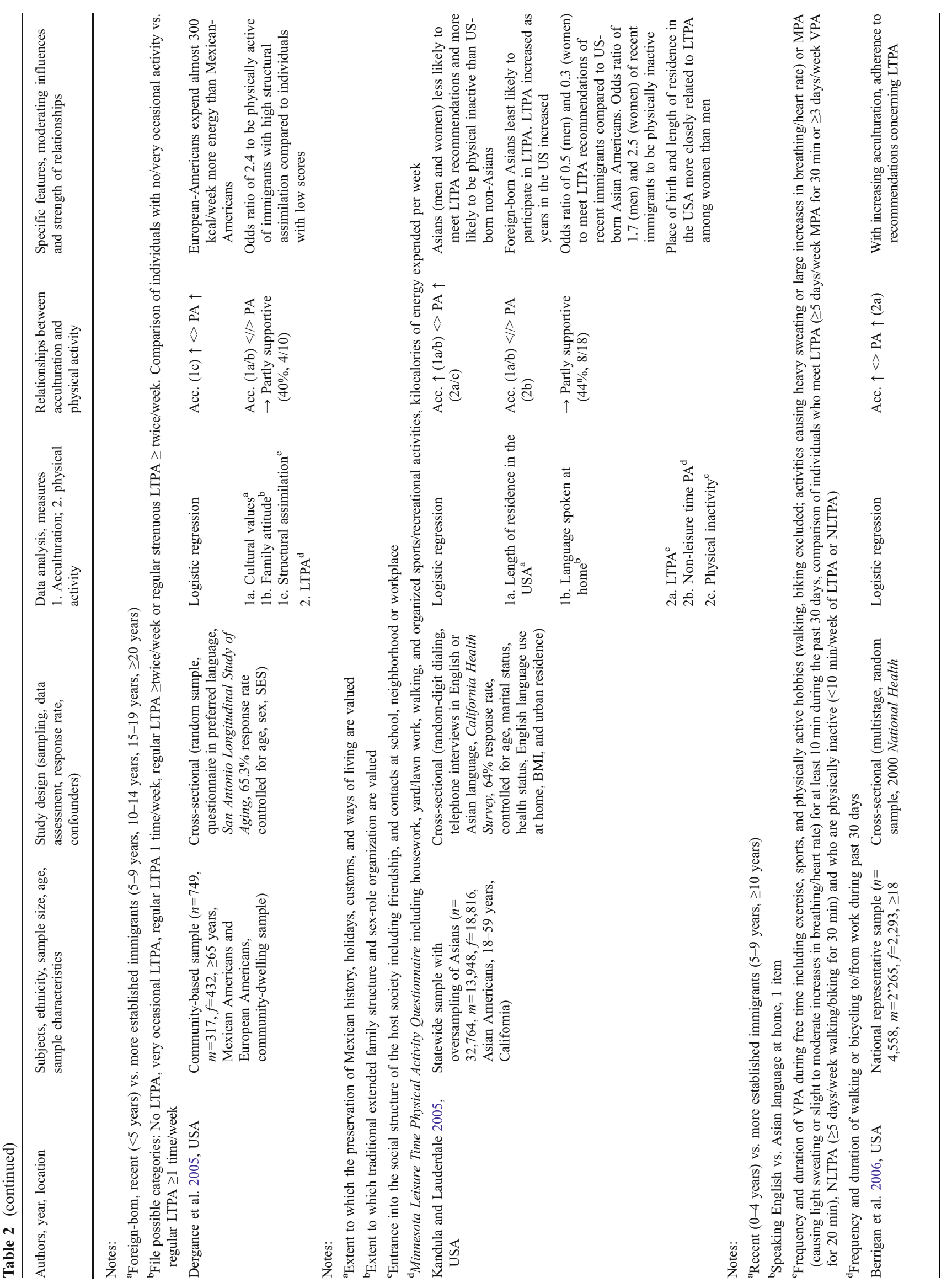




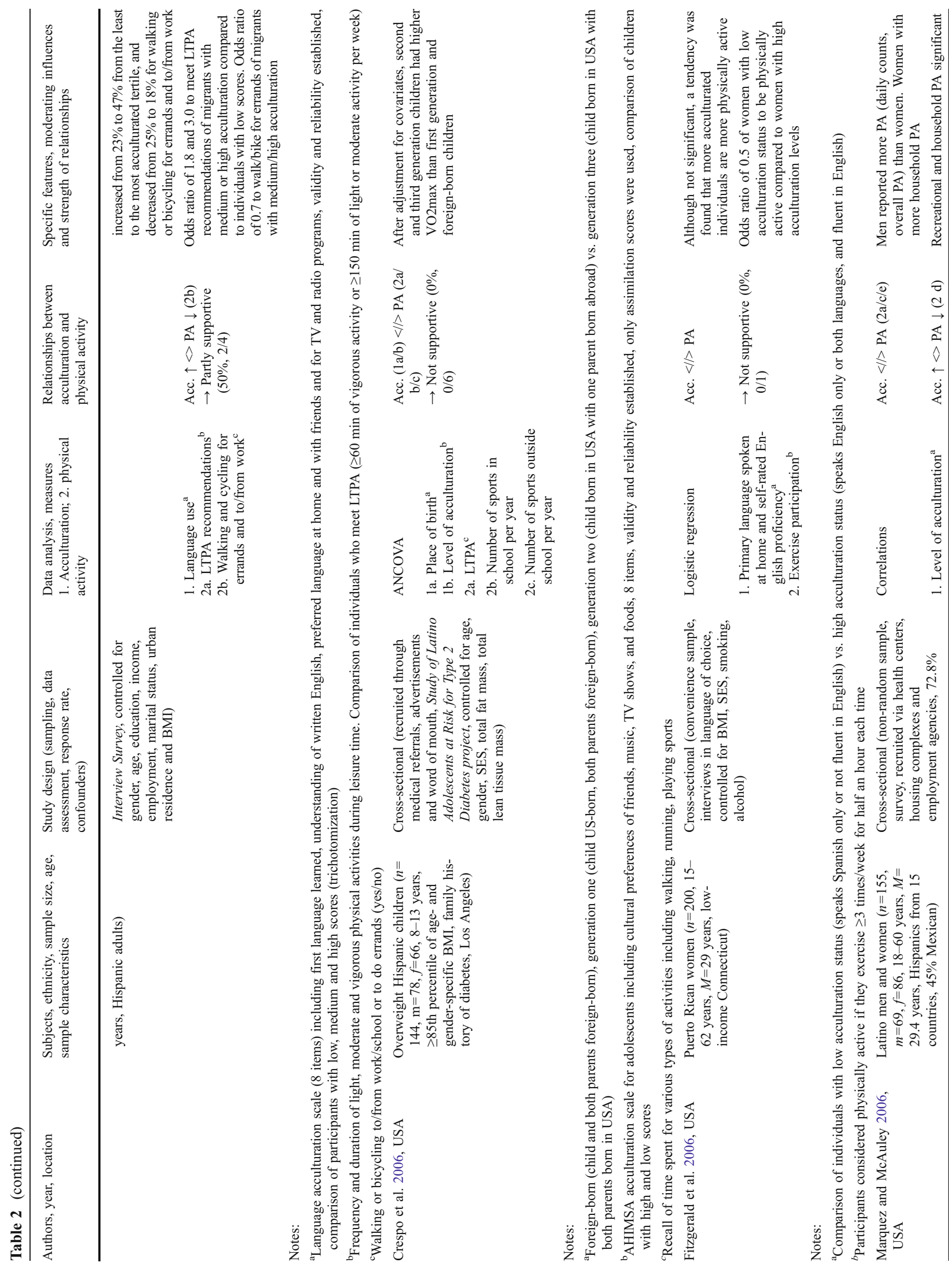




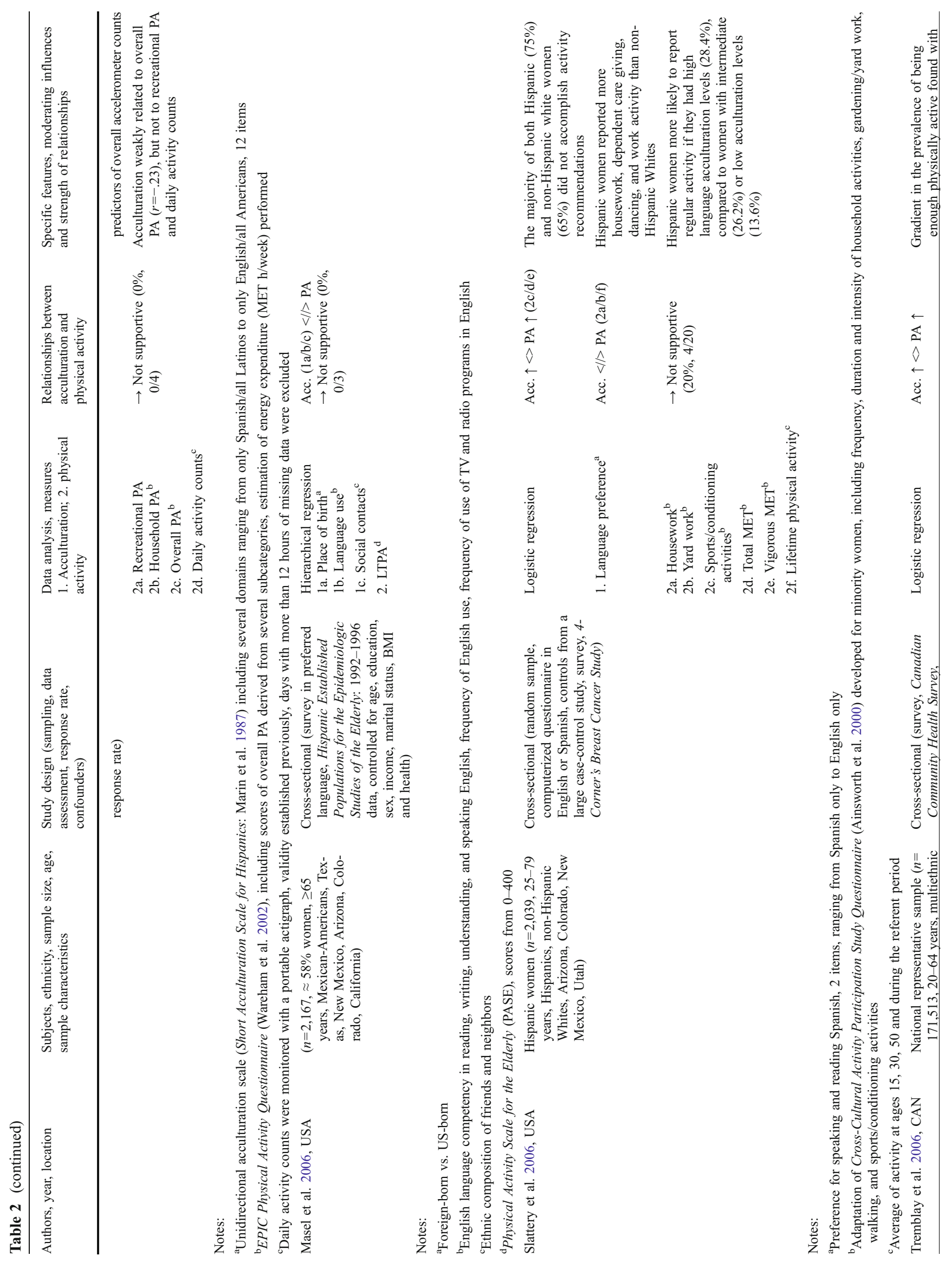




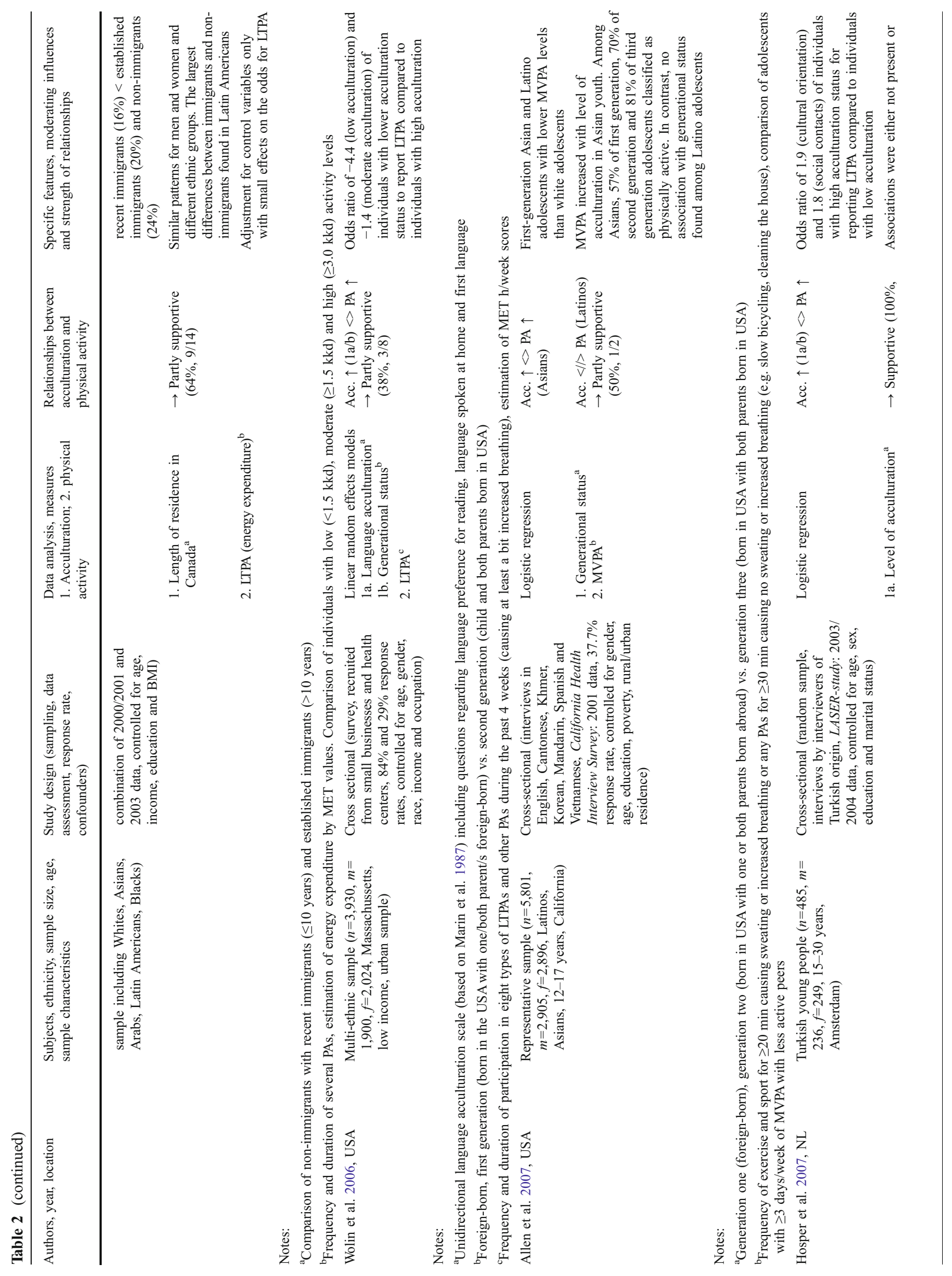









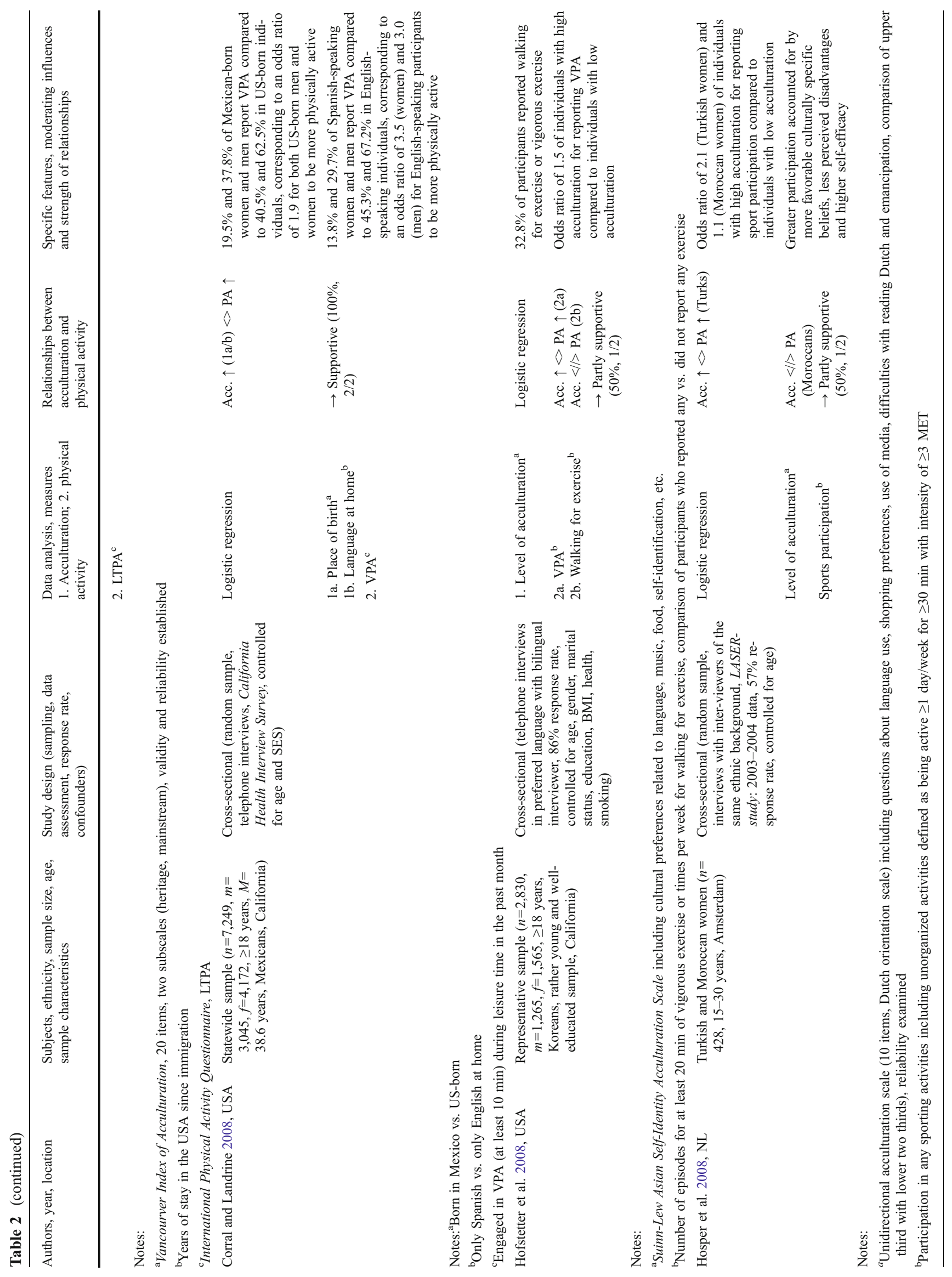




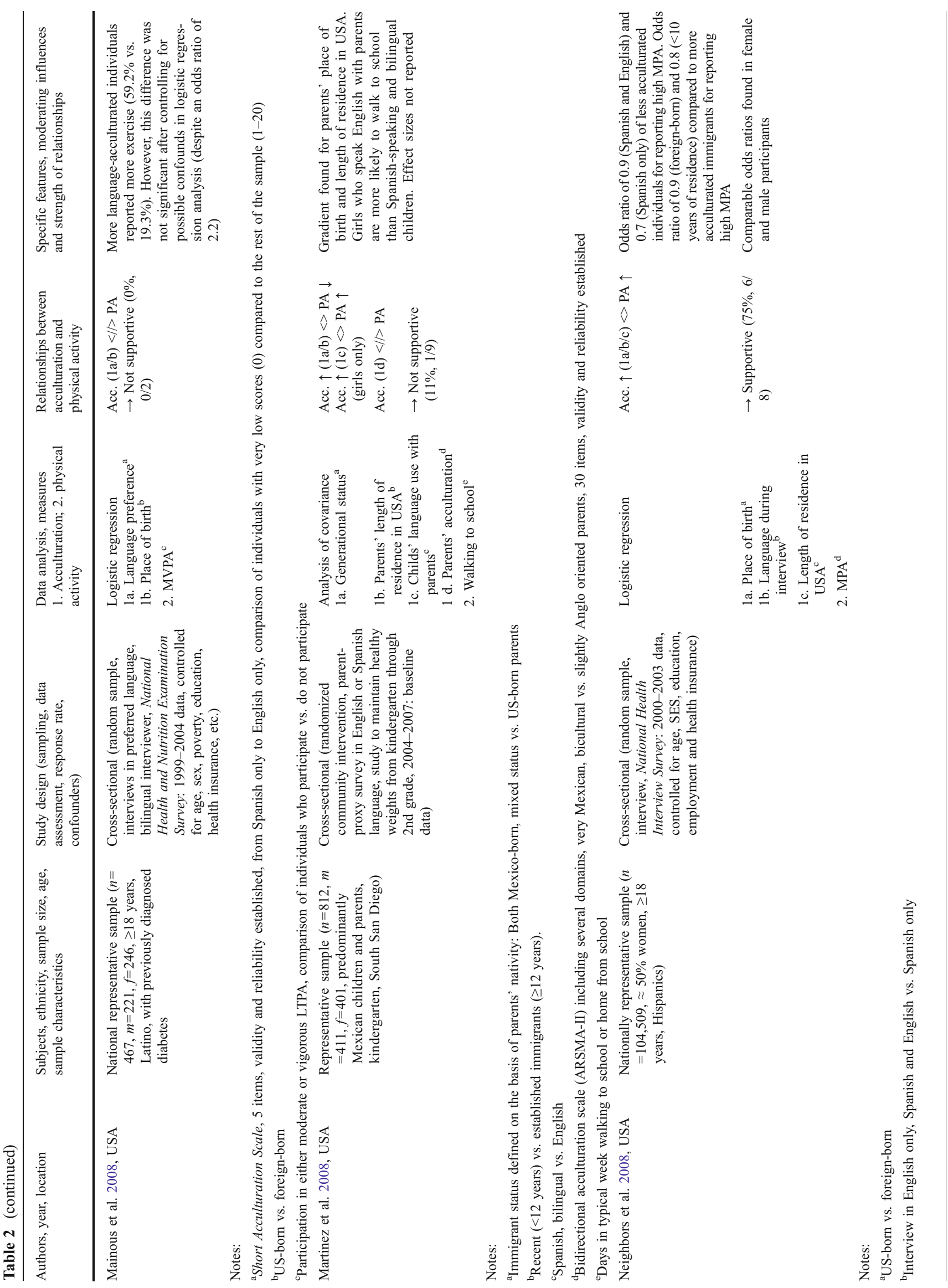









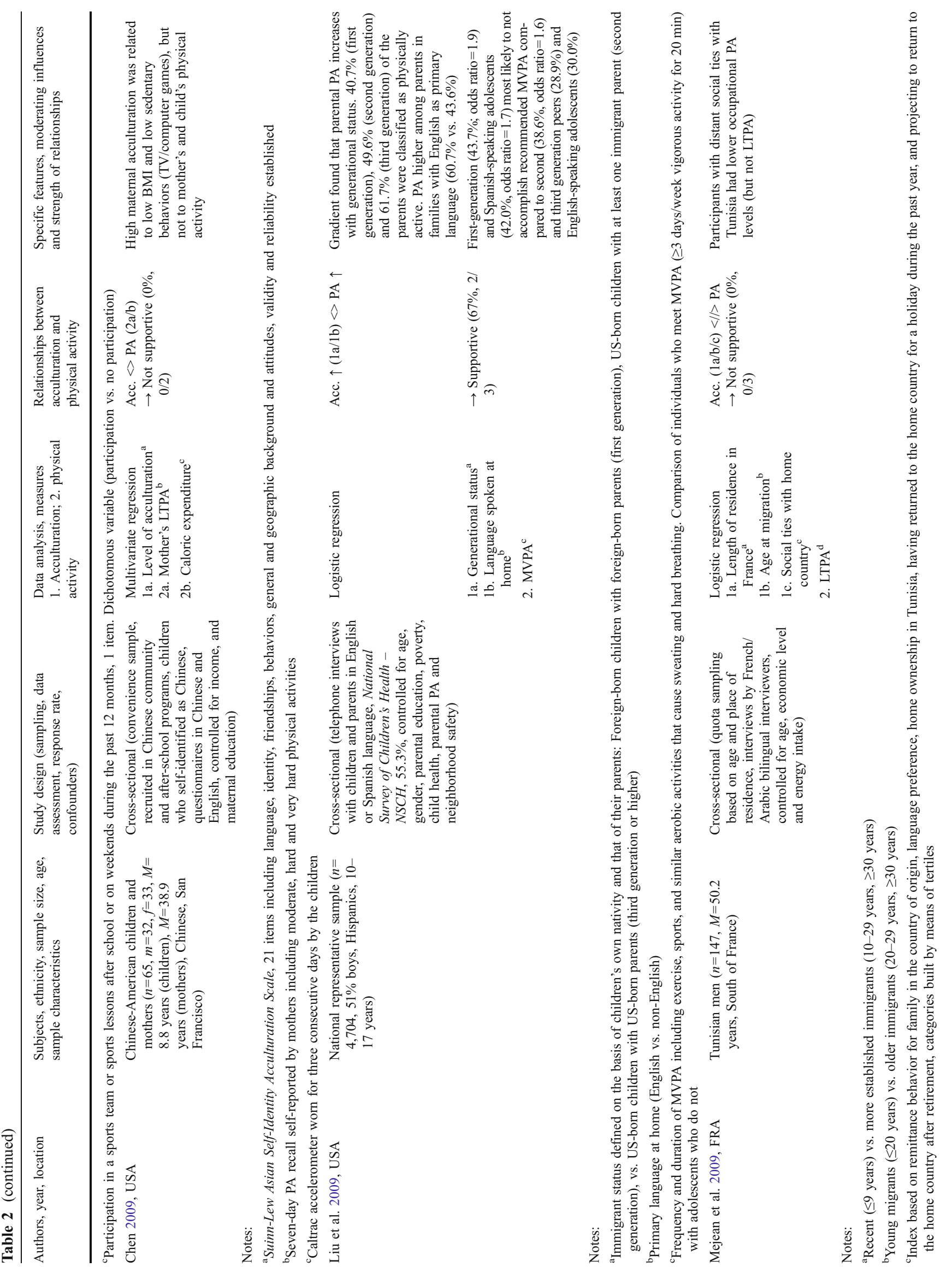




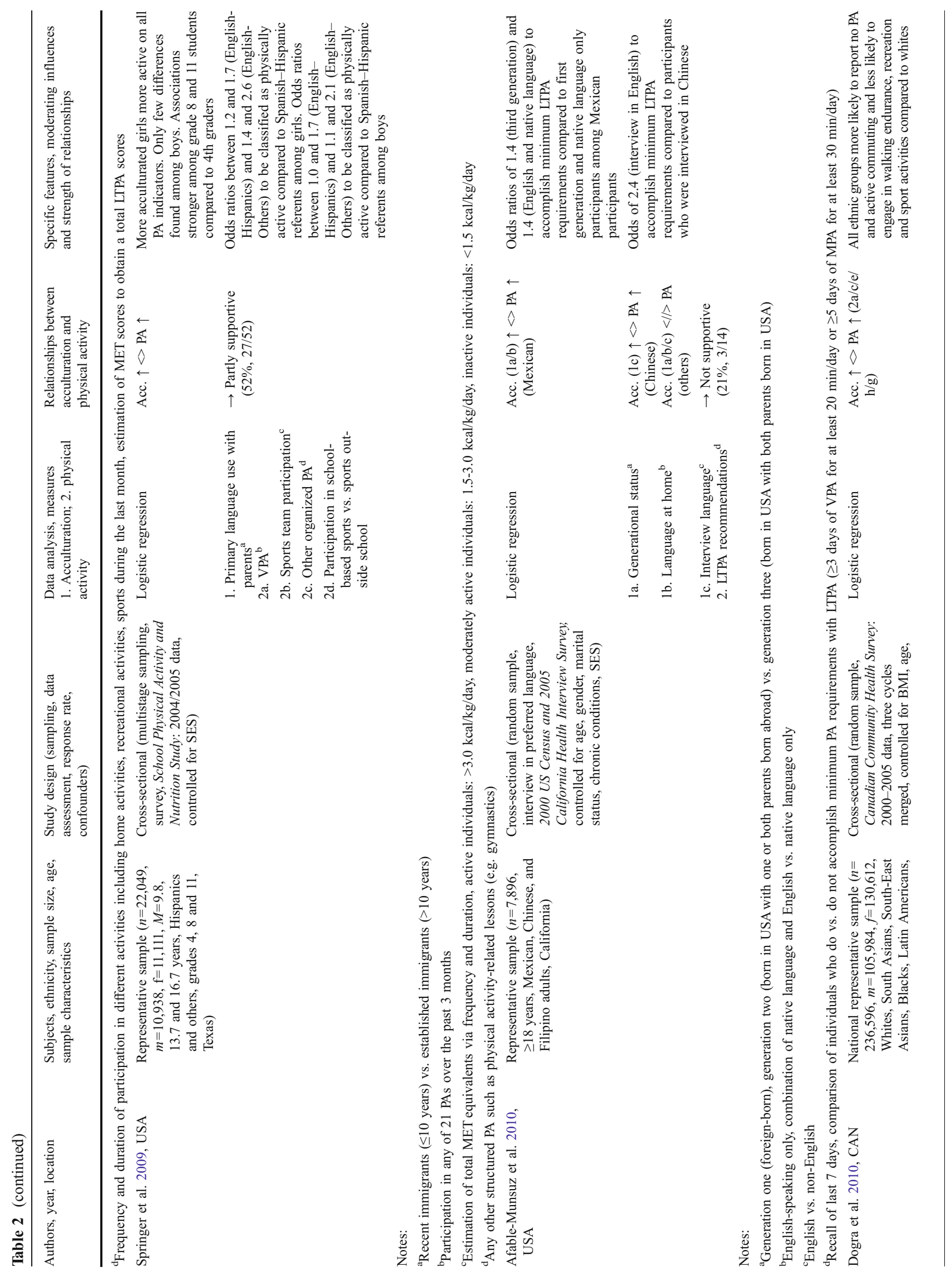




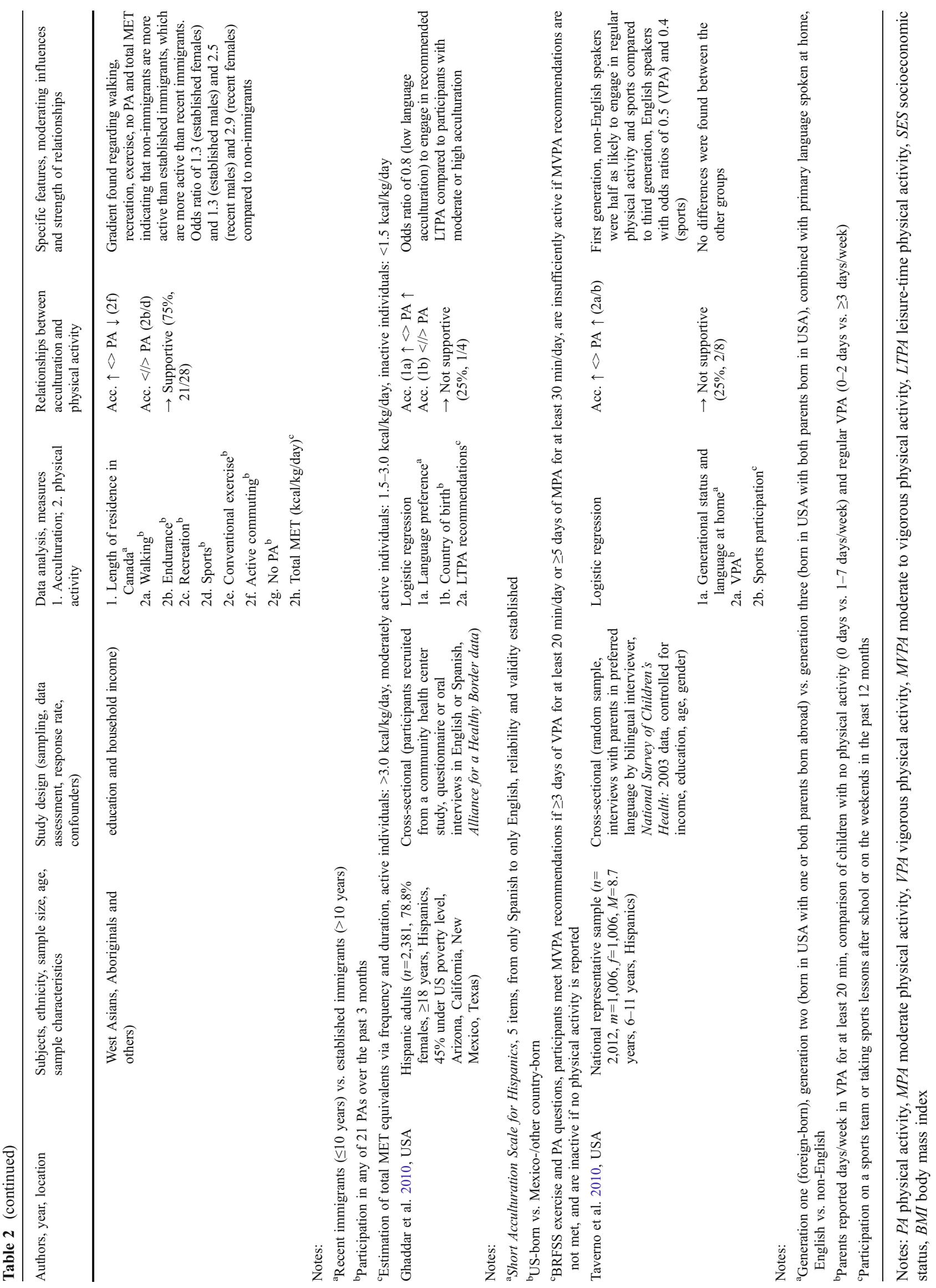


however, were conducted only in approximately half of the studies. Three investigations revealed a closer relationship between acculturation and physical activity among women than men (Abraido-Lanza et al. 2005; Dawson et al. 2005; Martinez et al. 2008).

Ethnicity The relationship between acculturation and physical activity has been most often examined with Hispanic samples (24 studies). Eight studies have focused on Asians. One study was limited to Sub-Saharan African Children (Renzaho et al. 2008). Several investigations used multiethnic samples (Dawson et al. 2005; Dogra et al. 2010; Tremblay et al. 2006; Wolin et al. 2006), or recruited individuals from several particular ethnic populations (Afable-Munsuz et al. 2010; Allen et al. 2007; Hosper et al. 2008; Singh et al. 2008; Unger et al. 2004). Some studies were confined to individuals with specific ethnic backgrounds such as Mexicans (Crespo et al. 2001; Dergance et al. 2005; Martinez et al. 2008; Masel et al. 2006; Wilbur et al. 2003), Puerto Ricans (Fitzgerald et al. 2006), Koreans (Choi et al. 2008; Hofstetter et al. 2008; Richman et al. 2000; Song et al. 2004), Turks (Hosper et al. 2007) and Tunisians (Mejean et al. 2009). Partly supporting results were found in Hispanic, Asian, Turkish, North and Sub-Saharan African, and multiethnic samples. Likewise, non-significant findings existed across all ethnic groups. This suggests that ethnic background does not moderate the association between acculturation and physical activity.

Date and source of publication The interest in this topic is fairly recent and started in the 1990s (Pérez-Stable et al. 1994). All studies selected for this review were published in peer-reviewed journals. That is, date and source of publication did not appear to moderate the relationship between acculturation and physical activity.

Sample size With respect to small-scale studies $(n<500)$, supportive and partly supportive findings were found in six investigations (Choi et al. 2008; Hosper et al. 2007; Hosper et al. 2008; Lee et al. 2000; Renzaho et al. 2008; Richman et al. 2000), while eight studies yielded insignificant relationships (Chen 2009; Crespo et al. 2006; Fitzgerald et al. 2006; Mainous et al. 2008; Marquez and McAuley 2006; Martinez et al. 2008; Wilbur et al. 2003; Yang et al. 2007). In studies with sample sizes between 500 and 1,000 participants, four studies were partly supportive $(67 \%)$, whereas two studies were not supportive $(33 \%)$. In contrast, of the studies with large sample sizes $(n>1,000)$, eight studies yielded supportive results, eight investigations provided partial support, whereas nine studies were not supportive. Thus, small samples appear to result in an underestimation of effects, while large sample sizes might lead to an overestimation.
Method of data analysis Logistic regression analysis was the most popular method of data analysis (used in 32 of 44 studies). Structural equation modelling (Pichon et al. 2007), linear random modelling (Wolin et al. 2006), linear regression analysis (Chen 2009; Masel et al. 2006; Unger et al. 2004; Yang et al. 2007), analysis of (co)variance (Crespo et al. 2006; Martinez et al. 2008; Richman et al. 2000), correlational analysis (Choi et al. 2008; Marquez and McAuley 2006; Richman et al. 2000) and chi-square testing (Cantero et al. 1999) were used less often. Interestingly, all (fully) supportive findings were based on studies using logistic regression analyses.

\section{Criterion 2: strength of association}

In logistic regression analyses, odds ratios provide an indication of the strength of the impact of acculturation on physical activity. While an odds ratio of 1 indicates the same likelihood of an event occurring across two groups, an odds ratio $>1$ implies that the event is more likely in the first group. In the literature reviewed, the highest effects were found in the study conducted by Lee et al. (2000) with odds ratios of 4.3 (men) and 7.4 (women) for traditional Korean Americans to report lower light physical activity compared to more acculturated individuals. Fairly high odds ratios $(>2.0)$ were found in other studies, too (Abraido-Lanza et al. 2005; Berrigan et al. 2006; Corral and Landrine 2008; Dergance et al. 2005; Dogra et al. 2010; Evenson et al. 2004; Hosper et al. 2008; Kandula and Lauderdale 2005; Pérez-Stable et al. 1994; Springer et al. 2009; Wolin et al. 2006). Most of the odds ratios ranged between 1.3 and 2.0 pointing towards a moderate association between acculturation and physical activity. Some studies did not provide odds ratios. Nevertheless, Tremblay et al. (2006) observed a gradient in the prevalence of being physically active showing that recent immigrants (16\%) less often reported high physical activity levels than established immigrants $(20 \%)$, which in turn were less active than non-immigrants (24\%).

\section{Criterion 3: specificity of effects}

Regarding the measurement of physical activity, support for our hypothesis comes predominantly from studies using general measures of moderate to vigorous physical activity including leisure and non-leisure activities such as exercise, sport, household and gardening activities. Regarding intensity of physical activity, Lee et al. (2000) highlighted that among Korean immigrants, more acculturated individuals reported higher levels of low intensity activity, whereas no relationships occurred for vigorous activities. Studies referring to established physical activity recommendations mostly evidenced partial support (Berrigan et al. 2006; 
Evenson et al. 2004; Kandula and Lauderdale 2005; Liu et al. 2009). Concerning sport and exercise participation, support and partial support was found in four studies (Dogra et al. 2010; Hosper et al. 2008; Singh et al. 2008; Springer et al. 2009), while insignificant findings occurred in six investigations (Cantero et al. 1999; Carvajal et al. 2002; Crespo et al. 2006; Fitzgerald et al. 2006; Slattery et al. 2006; Taverno et al. 2010). Specifically, Dogra et al. (2010) found that more acculturated immigrants invest more time in conventional exercise, while no main effect was found for general sport participation. Walking/biking (in the sense of active commuting) was negatively associated with acculturation in two studies (Berrigan et al. 2006; Dogra et al. 2010), while no significant relationships were substantiated in three investigations (Kandula and Lauderdale 2005; Martinez et al. 2008; Pichon et al. 2007). In contrast, walking as a leisure activity appeared to be positively related to acculturation (Dogra et al. 2010; Hofstetter et al. 2008).

Twenty-one studies included separate analyses for two or more acculturation measures, whereas the remaining investigations were limited to one specific indicator. Seven studies have explored the independent influence of participants' place of birth, while most of the research (15 studies) used length of residence as the independent variable. It is noteworthy that the categorization varied markedly between studies. For instance, while some researchers differentiated between immigrants who lived for more/less than 3 years in the USA (e.g. Evenson et al. 2004), others distinguished between individuals who have lived for more/less than 25 years in the host culture (e.g. Cantero et al. 1999), and yet others (e.g. Crespo et al. 2001) used narrower categories in order to examine linear gradients between immigrants with differing levels of acculturation (e.g. born in the host society, $<5$ years, 59 years, $10-19$ years vs. $\geq 20$ years). Eight studies used generational status as an indicator of acculturation. Language related aspects of acculturation were assessed in 20 investigations. Language use at home was applied nine times. Additionally, one study combined language use at home and language proficiency (Fitzgerald et al. 2006). Another nine studies used language preferences (often combined with usage). Most of the language preference scales consisted of several items asking about the first language learned, understanding of written English, preferred language at home, with friends or in general and language preference for TV and radio programs. Instruments that provide a broader description of the acculturation experience (e.g. behavioral and subjective elements of culture) and contain a bidimensional/orthogonal approach (independent orientation towards the original and new cultures) have been used less often. Nine studies included unidirectional measures of acculturation (Chen 2009;
Dergance et al. 2005; Hofstetter et al. 2008; Hosper et al. 2007; Hosper et al. 2008; Marquez and McAuley 2006; Pérez-Stable et al. 1994; Unger et al. 2004; Yang et al. 2007). Unger et al. (2004) used the AHIMSA acculturation scale, which draws on a bidirectional approach. However, only the US orientation scale was used to predict physical activity. Seven groups of researchers applied a bidirectional operationalization of acculturation (Carvajal et al. 2002; Choi et al. 2008; Lee et al. 2000; Martinez et al. 2008; Pichon et al. 2007; Renzaho et al. 2008; Song et al. 2004) such as the ARSMA-II scale.

In summary, no clear-cut patterns existed regarding the issue of which acculturation measure is most strongly associated with physical activity. Supportive and partly supportive findings were both found with more objective indicators (place of birth, length of residence, generational status), behavioral constructs (language use) and attitudinal variables (language preference, multidimensional acculturation).

\section{Criterion 4: temporality}

To date, only one study (Unger et al. 2004) with 11 year-old adolescents used a prospective design examining how acculturation in grade 6 predicts moderate to vigorous activity in grade 7 (although it did not control for baseline activity). This study did not support our primary hypothesis. The remaining findings were all based on crosssectional data.

\section{Criterion 5: gradient}

Few studies have used a design or strategies of data analyses that allowed the examination of a gradient. Crespo et al. (2001) showed that the odds ratio of less acculturated Mexicans for reporting physical inactivity increased from 1.3 to $1.5,1.4$ and 1.7 compared to US born individuals, depending on whether immigrants have lived $\geq 20$ years, 10-19 years, 5-9 years or $<5$ years in the USA. Similarly, Dawson et al. (2005) showed that among women (but not men) the odds ratios for no regular physical activity increased gradually depending on whether immigrants have lived 15-19 years (1.0), 10-14 years (1.4), 5-9 years (1.7) or $<5$ years (2.0) in Sweden compared to the most established referents $(\geq 20$ years). No gradient was found in the remaining studies (Mejean et al. 2009; Wilbur et al. 2003). Moreover, few studies offered combined analyses of two or more acculturation indicators. Fitzgerald et al. (2006) compared Puerto Rican women who spoke only Spanish or were not fluent in English with females who spoke English or were bilingual and who were fluent in English. Although not significant, an odds ratio of 0.5 to be physically active was found for women with low accultur- 
ation status compared to females with high scores. Taverno et al. (2010) combined generational status and language usage. Their findings showed that only first-generation immigrants who were non-English speakers had lower odds to report no regular physical activity. For second and third generation immigrants no differences were found as a function of their primary language used at home.

\section{Criterion 6: plausibility}

As shown in Table 2, most studies have been sufficiently controlled for confounding variables. Most studies controlled for age, gender and education. Moreover, a majority of studies included one measure of socioeconomic status such as employment status, income, or poverty. Less frequently used variables were marital status, number of children, household composition, language usage, urban/ rural residence, having a driving license, parental physical (in)activity, neighborhood attractiveness and safety, health, disability, health related fatalism, drinking, smoking or BMI. Several studies showed that the strength of the association between acculturation and physical activity decreased or became insignificant after controlling for sociodemographic influcences (Berrigan et al. 2006; Tremblay et al. 2006; Wolin et al. 2006).

Despite the fact that prior research has identified physical environment as a determinant of individuals' physical activity (e.g. Humpel et al. 2002), few studies have examined perceived environment as a moderating variable. Pichon et al. (2007) found that perceived neighborhood safety/aesthetics did not mediate between acculturation and physical activity. In contrast, Hosper et al. (2007) highlighted that the relationship between acculturation and physical activity was less strong among individuals who had children, lived in less attractive neighbourhoods and who reported high occupational physical activity.

\section{Criterion 7: experimental evidence}

To date, no experimental evidence exists showing that acculturation has an impact on individuals' physical activity levels.

\section{Discussion}

The main goal of the present review was to examine previous research dealing with the question of whether acculturation leads to changes in immigrants' physical activity levels. From a public health perspective, this issue is highly relevant as prior research has shown that (1) health practices of immigrants may deteriorate after arrival in the host society, and (2) that physical activity is a health- enhancing behavior that contributes to individuals' physical and mental well-being and prevents the development of chronic diseases such as cardiovascular diseases, cancer, osteoporosis, diabetes mellitus and obesity. Our hypothesis was that individuals would increase their non-occupational physical activity levels as they acculturate to the new society. This relationship would be in opposition to other health (damaging) behaviors such as smoking or alcohol intake, which seem to increase with augmenting acculturation (Pichon et al. 2007).

Nine studies provided support for our hypothesis, whereas 16 investigations were partly supportive. In contrast, 19 investigations were unable to detect the expected effects. In four of these studies, higher acculturation was related to decreased physical activity. In summary, the global analysis showed that $57 \%$ of all investigations at least partly supported our hypothesis of a positive relationship between daily and leisure time physical activity. With 19 non-supportive studies, a considerable variability was nonetheless found. This variability was not unexpected given the diversity of sample characteristics, study designs and measurements. Therefore, further criteria were taken into account to determine whether researchers have been successful in demonstrating causal evidence.

Besides the main hypothesis, seven study questions were formulated in relation to Hill's criteria of causal evidence (Hill 1965). That is, consecutive (moderator) analyses were conducted in order to gain deeper insight into the consistency of the findings, the strength of associations, the specificity of effects, issues of temporality, possible gradients, plausibility and experimental evidence. Each of these themes will be discussed separately in the following.

First, the associations are consistent in the sense that significant results were found both in North American and European research and among female and male immigrants. Cross-cultural consistency is important as the sport systems and the meanings ascribed to physical activity, exercise and sport may vary across different cultures (BrandlBredenbeck and Brettschneider 1997; De Knop 1998). With regard to gender, most studies on physical activity stress the differences between females and males - in a meta-analytic review of 108 studies, this difference was almost universal and persisted throughout adolescence and adulthood (Sallis et al. 2000). Regarding the process of acculturation, the literature is split on whether the adjustment period is more difficult for men or for women. Some authors claimed that the adaptation period is particularly strenuous for men who often are (or become) the sole breadwinners. Others argued that women more strongly feel the impacts of immigration as they might be forced to enter the job market for the first time in their lives (Stodolska and Alexandris 2004). As our review showed, acculturation seems to positively influence physical activity both among 
men and women. Furthermore, researchers have argued that it is easier for children to establish contacts and to assimilate, especially as children who attend mainstream schools have little choice but to interact with mainstream peers (Stodolska and Alexandris 2004). Thus, it was expected that age might impact on how acculturation influences health practices. However, the present review does not support that the associations between acculturation and physical activity depended on participants' age. Nevertheless, studies comparing different age groups are missing. Moreover, the association seemed independent of participants' ethnicity although prior research has shown considerable differences in physical activity related to participants' racial/ethnic background (e.g. Lindström and Sundquist 2001). With respect to methodological influences, studies with large sample sizes more often produced significant results. Sample size affects most tests of significance in the sense that using large samples increases the likelihood of finding significant relationships. Thus, odds ratios are more likely to be significant in large (e.g. $n$ $=1,000$ ) than small samples (e.g. $n=100$ ). Our review indicates that sample size moderates the relationship between acculturation and physical activity. Furthermore, all supportive findings were found in studies using logistic regression analyses, in which physical activity was treated as a categorical variable. Thus, even though in most cases information about frequency and duration of physical activity was available and would have allowed the calculation of metric scores, many researchers opted for transforming these values into categorical variables (often with the intention of differentiating between individuals who do/do not accomplish current physical activity recommendations). Perhaps, logistic regressions were best suited to finding support because the relationship between the two constructs is not linear. In summary, neither the host society, age, gender, ethnicity of the participants, the date nor the source of publication had a moderating impact. In contrast, studies with large sample sizes and using logistic regression analyses yielded more supportive findings.

The second question was related to the strength of the relationship between acculturation and physical activity. The odds ratios of more acculturated immigrants for being more physically active mainly ranged between 1.3 and 2.0, although higher odds were found in some studies. Taken together, the relationships between acculturation and physical activity were substantial, particularly as most analyses were controlled for multiple confounds such as age, gender, education and socioeconomic status.

Third, specificity of effects was not demonstrated as various measures of physical activity and acculturation yielded significant findings. To date, almost all findings are based on self-report measures. The only two studies using objective data (Chen and Wu 2008; Marquez and McAuley
2006) did not reveal significant relationships between acculturation and daily step counts. Moreover, the levels of physical activity that researchers considered as 'enough' physical activity varied considerably. For instance, Hosper et al. (2007) classified individuals as active if they engaged in more than $30 \mathrm{~min}$ of moderate activity per session at least 1 day/week. In comparison, Carvajal et al. (2002) considered participants as physically active if they have participated more than 4 days in heavy exercise or more than 5 days in lighter exercise. Thus, arbitrary cut-off criteria without reference to current physical activity recommendations complicate the comparison of the findings. Although the measurement of acculturation is still a matter of debate in public health literature, numerous scales are available to measure acculturation. The most commonly used indicators were place of birth/generational status, length of residence in the dominant culture, and language use/proficiency. More recently, these indexes were criticized as insufficient as they cannot capture individual nuances and differences in acculturation and assume a linear trend within the acculturation process. Thus, the underlying assumption of these measures is that adherence to values of the dominant culture increases with higher acculturation. However, this notion has been contested because not all immigrants adopt the values and norms of the majority culture even after a long period of residence in the host society (Stodolska and Alexandris 2004). Moreover, language use and proficiency do not necessarily reflect immigrants' adherence to the values and norms within the majority culture (Hosper et al. 2007), and individuals might acculturate with a different rate in various domains (Lara et al. 2005). Recently, researchers have addressed these issues by developing multidimensional scales tapping different domains such as ethnic identity, language, food, music preferences, and social affiliation with people from the minority or majority culture. The advocates of this approach claim that personal attitudes are key in the acculturation process and that the inclusion of different domains in which people acculturate provides a more grounded reflection of the acculturation process, than a one-sided and arbitrary focus on one specific indicator (e.g. Abraido-Lanza et al. 2006). In summary, the relationship between acculturation and physical activity did not depend on the instruments used to assess acculturation or physical activity. However, the review identified a need for studies, in which acculturation is operationalized in a really bidirectional way, and in which more objective measures are used to assess physical activity.

Fourth, temporality of the effects could not be established as 43 of 44 studies were cross-sectional. Despite the fact that studies using length of residence as an independent variable contain a temporal component, this construct cannot separate individual changes from cohort effects. 
Thus, in future studies, researchers should attempt to follow immigrants up over a extended period of time to find out whether increased lengths of residence, language use and the convergence of values towards the dominant culture are in fact accompanied by increased physical activity. Longitudinal research would also allow a more careful examination of mediators that explain how acculturation influences physical activity. In summary, with this limitation in mind, it cannot be determined from past research whether acculturation influences physical activity or vice versa, especially if language and multidimensional acculturation are used as predictor variables.

Fifth, a gradient showing that increased acculturation is paralleled by increases in physical activity was found in two studies. Finding a gradient, however, is complicated by the fact that some studies compared only two categories (Cantero et al. 1999; Dogra et al. 2010; Evenson et al. 2004; Tremblay et al. 2006). Studies looking at interactions between two or more indicators of acculturation (e.g. long time of residence combined with high language proficiency) are missing so far. In summary, the number of studies examining a gradient between acculturation and physical activity is too limited to establish such a relationship.

Sixth, to examine the plausibility of the findings, we have checked whether the analyses have been sufficiently controlled for social and demographic confounds, and whether environmental factors have been considered as potential influences. Controlling sociodemograhic factors is important as scientists have argued that acculturation may be a proxy for other variables. For instance, children in immigrant families are more likely than native-born children to be poor, to live in crowded housing, to be uninsured, to lack a source of health care, and to report poor health (Yu et al. 2003). Likewise, English language use is associated with increased parental education, child health, and neighborhood safety, as well as decreased poverty and parental physical inactivity (Liu et al. 2009). It is therefore noteworthy that all supportive studies were sufficiently adjusted for potential confounds. In contrast, attempts to get a better understanding of environmental influences that might moderate the relationship between acculturation and physical activity have been extremely sparse. One study showed that low perceptions of neighborhood safety canceled out the impact of acculturation (Hosper et al. 2007). In summary, previous studies were sufficiently controlled for social and demographic factors, and the findings of this review are based on the adjusted analyses. In contrast, information whether the relationship between acculturation and physical activity depends on environmental factors is largely missing.

Finally, the usefulness of experimental research in this area seems relatively small since acculturation processes are best analyzed in natural settings. With humans, manipulat- ing or training acculturation is difficult as programs to improve acculturation and integration might appear suspicious to immigrants who presume an assimilationist ideology behind such endeavors. Furthermore, given the tendency towards increasing individualism in Western societies, it remains questionable whether it is realistic to talk about a mainstream culture or if it is necessary to talk about mainstream cultures. Furthermore, changes in individuals' cultural identity develop slowly and might not occur before several years of residence in the host country. In contrast, experimentally manipulating exercise levels is possible. Thus, experimental research seems more suited to address the question of whether physical activity supports the acculturation process among immigrants.

Regarding the mechanisms responsible for association between acculturation and physical activity, several explanations have been offered. Pichon et al. (2007) noted that many recent immigrants arrive from rural or impoverished environments where physical activity might not be encouraged or valued (particularly in women). For instance, the Pan-European study examined attitudes and beliefs surrounding physical activity in 150,239 individuals in 15 countries and showed that Southern Europeans valued physical activity less than individuals living in other European countries (Kafatos et al. 1999). Thus, specific attitudes (e.g. exercise is for young people, exercise is more for men, exercise is only for people who are healthy or really good at it, exercise conflicts with religious beliefs) might prevent some immigrants from being more physically active (Hosper et al. 2008). Springer et al. (2009) for instance found in a study with Hispanic immigrants that cultural values related to modesty (i.e., feeling embarrassed by how one looks in gym attire), machismo (i.e., spouses/ partners do not want women to go out and exercise with other men), and familismo (i.e., importance of putting family needs before one's own needs, housework responsibilities) were important barriers preventing regular physical activity. Similarly, Kloetze (2001) reported that married Turkish women in the Netherlands only left the house when they could take their children with them due to responsibility for house and children.

Another underlying assumption is that health-related beliefs or norms concerning particular behaviors change with greater acculturation (Abraido-Lanza et al. 2006). Immigrant families may not be fully aware of physical and psychological health benefits of physical activity and sports participation (Dawson et al. 2005; Singh et al. 2008). Changes in health-related attitudes following immigration, however, have seldom been tested empirically (AbraidoLanza et al. 2006). Some researchers reported that perception of ideal body weight decreases among more acculturated Latinas (Ayala et al. 2007) and that the wish of having a slimmer figure may become a major motivator for 
physical activity. Another explanation assumes that exposure to and perception of health promotion campaigns is greater among people who speak the native language and who have many contacts with the host population (Hosper et al. 2007). Despite this, Unger et al. (2004) warn against the idea that attitudinal changes automatically lead to more healthy behaviors. Rather, adolescents might adopt a preference for activities and foods classified as "Western", including sedentary activities such as watching TV, playing video games, and eating fast-food. Furthermore, Crespo et al. (2001) lamented that health education materials emphasizing active lifestyles for immigrants are not culturally specific enough or readily available in the minority language.

Finally, researchers have suggested that immigrant parents may lack the time to transport children to structured activities, or the money to pay fees and acquire equipment (Liu et al. 2009), and that, in fact, immigrant parents may discourage their children's participation in sports because of fear of bullying, linguistic barriers, and the household employment situation (Liu et al. 2009; Yu et al. 2003). Additionally, immigrant parents may place higher value on children's time devoted to reading and learning activities, language lessons, academic performance, and participation in family activities. Safety concerns are another important consideration that may interact with gender-specific cultural values of heightened parent protectiveness for minority girls (Springer et al. 2009).

The findings of the present review must be interpreted with the following limitations in mind: Establishing a classification system to categorize studies into supportive, supportive and not supportive might seem arbitrary and the conclusion might depend on the categories chosen. Nevertheless, to provide a balanced interpretation, we have decided to classify studies into equally broad categories and to indicate the percentage of supportive findings for each investigation. Furthermore, sample size of the different studies was not weighted although this might rather lead to an under- than overestimation of the results (as studies with small sample sizes yielded fewer significant results). However, it is noteworthy that many studies used (very) large samples. Furthermore, possible publication biases (e.g. non-publication of insignificant findings) cannot be excluded. Moreover, the present review is based on a narrative method of synthesis with the 5\% level of significance as a cut-off point. However, Hill's (1965) criteria of causal evidence allowed a more balanced picture whether there exists support for a causal influence of acculturation on physical activity. Finally, most studies reviewed used proxy measures of acculturation that do not fully reflect an individual's experience and orientation during the acculturation process. The present study suggests several important avenues for future research. First of all, research should be extended beyond North America and Europe in order to find out whether acculturation leads to higher physical activity levels in non-Western societies as well. Moreover, researchers should implement prospective or longitudinal designs in order to be able to draw causal conclusions. It seems also desirable to use more objective measures to assess physical activity, to use broad and bidirectional measures of acculturation, and to compare different age groups using the same instruments. Moreover, the gradient between acculturation and physical activity, the interaction of two or more indicators of acculturation, and the factors underlying the association between acculturation and physical activity need further examination.

\section{Conclusion}

The results of this review show that nine studies support a positive relationship between acculturation, whereas 19 studies did not find supporting evidence, and 16 investigations provided mixed results. Although $43 \%$ of all studies were not able to show significant relationships, the majority of the studies provided at least partly supportive findings. Thus, the findings suggest that efforts to promote acculturation (e.g. language learning) among immigrants might have positive outcomes regarding their levels of physical activity. Additionally, the present findings indicate that intervention programs aimed at stimulating participation in physical activity among immigrants and ethnic minority groups could give priority to those individuals with low acculturation levels (Hosper et al. 2008). For instance, messages promoting physical activity could be tailored to less acculturated immigrants and delivered via channels accessed by these populations including minority-language newspapers, magazines, television, and radio stations (Pichon et al. 2007). Moreover, culturally specific beliefs, needs and constraints could be taken into account when providing activity counseling (Crespo 2000; Hosper et al. 2008). Nevertheless, evidence regarding the effectiveness of such culturally tailored interventions is not established yet. It is our hope that this review will provide the foundation for and stimulate further research on the relationship between acculturation and physical activity.

Conflict of interest statement All authors declare that they have no conflict of interest.

\section{References}

Abraido-Lanza AF, Chao MT, Florez KR (2005) Do healthy behaviors decline with greater acculturation? Implications for the Latino mortality paradox. Soc Sci Med 61:1243-1255 
Abraido-Lanza AF, Armbrister AN, Florez KR, Aguirre AN (2006) Toward a theory-driven model of acculturation in public health research. Am J Public Health 96:1342-1346

Afable-Munsuz A, Ponce NA, Rodriguez M, Perez-Stable EJ (2010) Immigrant generation and physical activity among Mexican, Chinese \& Filipino adults in the US. Soc Sci Med 70:1997-2005

Ainsworth BE, Haskell WL, Whitt MC, Irwin ML, Swartz AM, Strath SJ, et al. (2000) Compendium of physical activities: an update of activity codes and MET intensities. Med Sci Sports Exercise 32: S498-S516

Allen ML, Elliott MN, Morales LS, Diamant AL, Hambarsoomian K, Schuster MA (2007) Adolescent participation in preventive health behaviors, physical activity, and nutrition: differences across immigrant generations for Asians and Latinos compared with whites. Am J Public Health 97:337-343

Ayala GX, Mickens L, Galindo P, Elder JP (2007) Acculturation and body image perception among Latino youth. Ethn Health 2007:21-41

Berrigan D, Dodd K, Troiano RP, Reeve BB, Ballard-Barbash R (2006) Physical activity and acculturation among adult Hispanics in the United States. Res Q Exerc Sport 77:147-157

Berry JW (1970) Marginality, stress and ethnic identification in an acculturated Aboriginal community. J Cross-Cultur Psychol $1: 239-252$

Berry JW (2006) Stress perspectives on acculturation. In: Sam DL, Berry JW (eds) The Cambridge handbook of acculturation psychology. University of Cambridge Press, Cambridge, UK, pp 43-57

BFS (2006) Bewegung, Sport, Gesundheit. Fakten und Trends aus den Schweizerischen Gesundheitsbefragungen 1992, 1997, 2002. Bundesamt für Statistik, Neuchâtel, Germany

Brandl-Bredenbeck H-P, Brettschneider W-D (1997) Sport involvement and self-concept in German and American Adolescents. Int Rev Sociol Sport 32:357-371

Byrd-Williams C, Kelly LA, Davis JN, Spruijt-Metz D, Goran MI (2007) Influence of gender, BMI and Hispanic ethnicity on physical activity in children. Int J Pediatr Obes 2:159-166

Cantero PJ, Richardson JL, Baezconde-Garbanati L, Marks G (1999) The association between acculturation and health practices among middle-aged and elderly Latinas. Ethn Dis 9:166-180

Carvajal SC, Hanson CE, Romero AJ, Coyle KK (2002) Behavioral risk factors and protective factors in adolescents: a comparison of Latinos and non-Latino whites. Ethn Health 7:181-193

Cuellar I, Arnold B, Maldonado R (1995) Acculturation rating scale for Mexican Americans, II: a revision of the original ARSMA scale. Hispanic J Behav Sci 17:275-304

Chen J-L (2009) Household income, maternal acculturation, maternal education level and health behaviors of Chinese-American children and mothers. J Immigr Minor Health 11:198-204

Chen J-L, Wu Y (2008) Cardiovascular risk factors in Chinese American children: associations between overweight, acculturation, and physical activity. J Pediatr Health Care 22:103-110

Choi JW, Wilbur J, Miller A, Szalacha L, McAuley E (2008) Correlates of leisure-time physical activity in Korean immigrant women. West J Nurs Res 30:620-638

Corral I, Landrine H (2008) Acculturation and ethnic-minority health behavior: a test of the operant model. Health Psychol $27: 737-745$

Crespo CJ (2000) Encouraging physical activity in minorities: eliminating disparities by 2010. Phys Sports Med 28:36-51

Crespo CJ, Smit E, Andersen RE, Ainsworth BE, Carter-Pokras O (2000) Race/ethnicity, social class and their relationship to physical inactivity during leisure time: results from the Third National Health and Nutrition Examination Survey. Am J Prev Med 18:46-53
Crespo CJ, Smit E, Carter-Pokras O, Andersen R (2001) Acculturation and leisure-time physical inactivity in Mexican American adults: results from NHANES III, 1988-1994. Am J Public Health 91:1254-1257

Crespo NC, Ball GDC, Shaibi GQ, Cruz ML, Weigensberg MJ, Goran MI (2006) Acculturation is associated with higher VO2 max in overweight Hispanic children. Pediatr Exerc Sci 18:89-100

Dawson AJ, Sundquist J, Johansson SE (2005) The influence of ethnicity and length of time since immigration on physical activity. Ethn Health 10:293-309

De Knop P (1998) Worldwide trends in youth sport. In: Naul R (ed) Physical activity and active lifestyle of children and youth. Hofmann, Schorndorf, Germany, pp 52-62

De La Rosa M, Vega R, Radisch MA (2000) The role of acculturation in the substance abuse behavior of African-American and Latino adolescents: advances, issues, and recommendations. J Psychoactive Drugs 32:33-42

Dergance JM, Mouton CP, Lichtenstein MJ, Hazuda HP (2005) Potential mediators of ethnic differences in physical activity in older Mexican Americans and European Americans: results from the San Antonio Longitudinal Study of Aging. J Am Geriatr Soc 53:1240-1247

Dogra S, Meisner BA, Ardern CI (2010) Variation in mode of physical activity by ethnicity and time since immigration: a cross-sectional analysis. Int J Behav Nutr Phys Act 7:2-11

Epstein JF, Sugerman SB, Mitchell P, Induni M (2008) Prevalence of fruit and vegetable consumption and physical activity by race/ ethnicity: California, 2005-2006. Californian J Health Promotion 6:61-72

Evenson KR, Sarmiento OL, Tawney KW, Macon ML, Ammerman AS (2003) Personal, social, and environmental correlates of physical activity in North Carolina Latina immigrants. Am J Prev Med 25:77-85

Evenson KR, Sarmiento OL, Ayala GX (2004) Acculturation and physical activity among North Carolina Latina immigrants. Soc Sci Med 59:2509-2522

Faulkner GEJ, Taylor AH (eds) (2005) Exercise, health and mental health. Routledge, London

Fitzgerald N, Himmelgreen D, Damio G, Segura-Perez S, Peng Y-K, Perez-Escamilla R (2006) Acculturation, socioeconomic status, obesity and lifestyle factors among low-income Puerto Rican women in Connecticut, U.S., 1998-1999. Pan Am J Public Health 19:306-313

Ghaddar S, Brown CJ, Pagan JA, Diaz V (2010) Acculturation and healthy lifestyle habits among Hispanics in United States-Mexico border communities. Pan Am J Public Health 28:190-197

Gordon-Larsen P, Adair LS, Popkin BM (2002) Ethnic differences in physical activity and inactivity patterns and overweight status. Obes Res 10:141-149

Gordon-Larsen P, Mullan Harris K, Ward DS, Popkin BM (2003) Acculturation and overweight-related behaviors among Hispanic immigrants to the US: The national longitudinal study of adolescent health. Soc Sci Med 57:2023-2034

Hill AB (1965) The environment and disease: association or causation? Proc R Soc Med 58:295-300

Hofstetter CR, Irvin V, Schmitz K, Hovell MF, Nichols J, Kim HR et al (2008) Demography of exercise among Californians of Korean descent: a cross-sectional telephone survey. J Immigr Minor Health 10:53-65

Hosper K, Klazinga NS, Stronks K (2007) Acculturation does not necessarily lead to increased physical activity during leisure time: a cross-sectional study among Turkish young people in the Netherlands. BMC Public Health 7:230

Hosper K, Nierkens V, van Valkengoed I, Stronks K (2008) Motivational factors mediating the association between accultur- 
ation and participation in sport among young Turkish and Moroccan women in the Netherlands. Prev Med 47:95-100

Humpel N, Owen N, Leslie E (2002) Environmental factors associated with adults' participation in physical activity: a review. Am J Prev Med 22:188-199

Kafatos A, Manios Y, Markatji I, Giachetti I, Vaz de Almeida M, Engström L (1999) Regional, demographic and national influences on attitudes and beliefs with regard to physical activity, body weight and health in a nationally representative sample in the European Union. Public Health Nutr 2:87-95

Kandula NR, Lauderdale DS (2005) Leisure time, non-leisure time, and occupational physical activity in Asian Americans. Ann Epidemiol 15:257-265

Kloetze JWR (2001) Integration through leisure? Leisure time activities and the integration of Turkish families in Arnhem and Enschede in the Netherlands. World Leisure 1:52-61

Lara M, Gamboa C, Iya Kahramanian M, Morales LS, Hayes Bautista DE (2005) Acculturation and Latino health in the United States: a review of the literature and its sociopolitical context. Annu Rev Public Health 26:367-397

Lee S, Sobal J, Frongillo E Jr (2000) Acculturation and health in Korean Americans. Soc Sci Med 51:159-173

Lindström M, Sundquist J (2001) Immigration and leisure-time physical inactivity: a population-based study. Ethn Health 6:771-778

Liu J, Probst JC, Harun N, Bennett KJ, Torres ME (2009) Acculturation, physical activity, and obesity among Hispanic adolescents. Ethn Health 14:509-525

Mainous AG, Diaz VA, Geesey ME (2008) Acculturation and healthy lifestyle among Latinos with diabetes. Ann Fam Med 6:131-137

Marin G, Gamba R (1996) A new measurement of acculturation for Hispanics: the Bidimensional Acculturation Scale for Hispanics (BAS). Hispanic J Behav Sci 18:297-316

Marin G, Sabogal R, Marin BV, Otero-Sabogal R, Perez-Stable EJ (1987) Development of a short acculturation scale for Hispanics. Hispanic J Behav Sci 9:183-205

Marquez DX, McAuley E (2006) Gender and acculturation influences on physical activity in Latino adults. Ann Behav Med 31:138144

Martinez SM, Ayala GX, Arredondo EM, Finch B, Elder J (2008) Active transportation and acculturation among Latino children in San Diego County. Prev Med 47:313-318

Masel MC, Rudkin LL, Peek MK (2006) Examining the role of acculturation in health behaviors of older Mexican Americans. Am J Health Behav 30:684-699

Mejean C, Traissac P, Eymard-Duvernay S, Delpeuch F, Maire B (2009) Influence of acculturation among Tunisian migrants in France and their past/present exposure to the home country on diet and physical activity. Public Health Nutr 12:832-841

Moher D, Liberati A, Tetzlaff J, Altman DG (2009) The PRISMA Group (2009). Preferred reporting items for systematic reviews and meta-analyses: the PRISMA Statement. PLoS Med 6: e1000097. doi:1000010.1001371/journal.pmed1000097

Neighbors CJ, Marquez DX, Marcus BH (2008) Leisure-time physical activity disparities among Hispanic subgroups in the United States. Am J Public Health 98:1460-1464

Pérez-Stable EJ, Marin G, VanOss MB (1994) Behavioral risk factors: a comparison of Latinos and non-Latino whites in San Francisco. Am J Public Health 84:971-976

Pichon LC, Arredondo EM, Roesch S, Sallis JF, Ayala GX, Elder JP (2007) The relation of acculturation to Latinas' perceived neighborhood safety and physical activity: a structural equation analysis. Ann Behav Med 34:295-303

Renzaho AMN, Swinburn B, Burns C (2008) Maintenance of traditional cultural orientation is associated with lower rates of obesity and sedentary behaviours among African migrant children to Australia. Int J Obes 32:594-600

Richman RM, Bermingham M, Ko JR, Mahajan D, Steinbeck KS, Caterson ID (2000) Cardiovascular risk factors in elderly Koreans in Australia and Korea. Asia Pac J Clin Nutr 9:46-52

Sagatun A, Kolle E, Anderssen SA, Thoresen M, Sogaard AJ (2008) Three-year follow-up of physical activity in Norwegian youth from two ethnic groups: associations with socio-demographic factors. BMC Public Health 8:419

Sallis JF, Owen N (1999) Physical activity and behavioral medicine. Sage, Thousand Oaks, CA

Sallis JF, Prochaska JJ, Taylor AC (2000) A review of correlates of physical activity of children and adolescents. Med Sci Sports Exerc 32:963-975

Sam DL (2006) Acculturation: conceptual background and core components. In: Sam LD, Berry JW (eds) The Cambridge handbook of acculturation psychology. Cambridge University Press, Cambridge, pp 11-26

Schoenborn CA, Adams PF, Barnes PM, Vickerie JL, Schiller JS (2004) Health behaviors of adults: United States 1999-2001. Vital and Health Statistics. Series 10: Data from the National Health Survey 219:1-79

Singh GK, Yu SM, Siapush M, Kogan MD (2008) High levels of physical activity and sedentary behaviors among US immigrant children and adolescents. Arch Pediatr Adol Med 162:756-763

Slattery ML, Sweeney C, Edwards S, Herrick J, Murtaugh M, Baumgartner K et al (2006) Physical activity patterns and obesity in Hispanic and non-Hispanic white women. Med Sci Sport Exer 38:33-41

Song YJ, Hofstetter CR, Hovell MF, Paik HY, Park HR, Lee J et al (2004) Acculturation and health risk behaviors among Californians of Korean descent. Prev Med 39:147-156

Springer AE, Lewis K, Kelder SH, Fernandez ME, Barroso CS, Hoelscher DM (2009) Physical activity participation by parental language use in 4th, 8th, and 11th grade students in Texas, USA. J Immigr Minor Health 12:769-780

Stodolska M, Alexandris K (2004) The role of recreational sport in the adaptation of first generation immigrants in the United States. J Leisure Res 36:379-413

Taverno SE, Rollins BY, Francis LA (2010) Generation, language, body mass index, and activity patterns in Hispanic children. Am J Prev Med 38:145-153

Tremblay MS, Bryan SN, Perez CE, Ardern CI, Katzmarzyk PT (2006) Physical activity and immigrant status: evidence from the Canadian Community Health Survey. Can J Public Health 97:277-282

Unger JB, Gallaher P, Shakib S, Ritt-Olson A, Palmer PH, Johnson CA (2002) The AHIMSA Acculturation Scale: a new measure of acculturation for adolescents in a multicultural society. J Early Adolescence 22:225-251

Unger JB, Reynolds K, Shakib S, Spruijt-Metz SP, Johnson A (2004) Acculturation, physical activity, and fast-food consumption among Asian-American and Hispanic adolescents. J Comm Health 29:467-481

Wareham NJ, Jakes RW, Rennie KL, Mitchell J, Hennings S, Day NE (2002) Validity and repeatability of the EPIC-Norfolk Physical Activity Questionnaire. Int J Epidemiol 31:168-174

Wendel-Vos GC, Schuit AJ, Saris WH, Kromhout D (2003) Reproducibility and relative validity of the short questionnaire to assess health-enhancing physical activity. J Clinical Epidemiol 56:11631169

Wilbur J, Chandler PJ, Dancy B, Lee H (2003) Correlates of physical activity in urban midwestern Latinas. Am J Prev Med 2003:S69 S76 
Wolin KY, Colditz G, Stoddard AM, Emmons KM, Sorensen G (2006) Acculturation and physical activity in a working class multiethnic population. Prev Med 42:266-272

Yang K, Laffrey SC, Stuifbergen A, Im EO, May K, Kouzekanani K (2007) Leisure-time physical activity among midlife Korean immigrant women in the US. J Immigr Minor Health 9:291-298
Yeager K, Macera CA, Merritt R (1993) Socioeconomic influences on leisure-time sedentary behavior among women. Health Values $17: 51-54$

Yu SM, Huang ZJ, Schwalberg RH, Overpeck M, Kogan MD (2003) Acculturation and the health and well-being of U.S. immigrant adolescents. J Adolesc Health 33:479-488 